\title{
OTULIN is a New Target for EA Treatment in Alleviating Brain Injury and The Activation of Glial Cells By Depressing NF-kB Signalling Pathway in Acute Ischaemic Stroke Rats
}

\section{Hongbei Xu}

The First Affiliated Hospital of Chongqing Medical University

\section{You Wang}

The First Affiliated Hospital of Chongqing Medical University

\section{Yong Luo ( 1023158588@qq.com)}

The First Affiliated Hospital of Chongqing Medical University Department of Neurology https://orcid.org/0000-0002-7739-5229

\section{Research article}

Keywords: Acute ischaemic stroke, Electroacupuncture, OTULIN, NF-KB signalling pathway, Brain injury, Glial activation

Posted Date: January 28th, 2021

DOl: https://doi.org/10.21203/rs.3.rs-153447/v1

License: (c) (i) This work is licensed under a Creative Commons Attribution 4.0 International License. Read Full License

Version of Record: A version of this preprint was published at Molecular Medicine on April 9th, 2021. See the published version at https://doi.org/10.1186/s10020-021-00297-0. 


\section{Abstract}

Objective: Ovarian tumour domain deubiquitinase with linear linkage specificity (OTULIN), a potent negative regulator of nuclear factor-KB (NF-KB) signalling pathway, exerted strong neuroprotective role following acute ischemic stroke. Electroacupuncture $(E A)$ is an effective adjuvant treatment for reducing brain injury and neuroinflammtion through inhibiting the nuclear translocation of NF-KB p65, while the underlying mechanism remains unclear. This study investigated whether OTULIN was necessary for EA to mitigate brain injury and the activation of glial cells, following by a transient middle cerebral artery occlusion (tMCAO) model in rats.

Methods: Acute ischaemic stroke model was established by performing tMCAO surgery in SpragueDawley (SD) rats. EA was performed once daily at "Baihui (GV 20)", "Hegu (LI 4)", and "Taichong (LR 3)" acupoints. EA's effect on the spatiotemporal expression of OTULIN in the ischaemic penumbra of cerebral cortex was detected within $7 \mathrm{~d}$ after reperfusion. Following gene interference to silence OTULIN expression, the effects of OTULIN on EA neurological deficits, cerebral infarct volume, neuronal damage, the activation of microglia and astrocytes, the contents of tumor necrosis factor alpha (TNF- $a$ ), interleukin-1 beta (IL-1 $\beta$ ) and interleukin-6 (IL-6), and the expression of $\mathrm{p}-\mathrm{IKBa}$, IKBa and nucleus/cytoplasm NF-KB p65 protein were assessed.

Results: EA treatment increased endogenous OTULIN expression, with a peak at $48 \mathrm{~h}$. Enhanced OTULIN was mainly located in neurons, and little OTULIN was detected in microglia as well. OTULIN silencing obviously reversed EA neuroprotection as demonstrated by worsened neurobehavioural performance, cerebral infarct volume and neuronal injury. Besides, the inhibitory effect of EA on NF-KB pathway was also attenuated by enhanced IKBa phosphorylation and NF-KB p65 nuclear translocation. Furthermore, EA partly inhibited the transformation of microglia and astrocytes to from resting states to activated states, and reduced the secretion of TNF- $a$, IL-1 $\beta$ and IL-6, but this preventive effects were reversed after silencing OTULIN expression.

Conclusions: OTULIN provides a new potential therapeutic target for EA to alleviate acute ischaemic stroke induced brain injury and the activation of glial cells, which is related to depression of NF-KB signalling pathway.

\section{Introduction}

Ischaemic stroke induced cell injury initiates the release of danger signals that activate the immune system in central nervous system (CNS)(Corps, Roth et al., 2015). The subsequent sterile inflammatory response mainly involves the innate immune system with activation of resident immune cells of the central nervous system (CNS) and a rapid infiltration of peripheral immune cells(Lin, Wang et al., 2020; Liu, Zhao et al., 2020; Shan, Zang et al., 2020; Wang, Xiong et al., 2020). Nuclear factor-kappa B (NF-KB) signalling pathway has been considered a "master regulator", as it dominates the initiation and development of brain injury induced by cerebral ischaemia(Kaushal and Schlichter, 2008; Liu, Zhang et 
al., 2017). Physically, the p65/p50 subunit dimer, the main form of NF-KB, is mainly located in the cytoplasm and combines with NF-KB inhibitor protein a (IKBa) in a resting form. Following activation by upstream kinases after ischaemia onset, IKBa is degraded and phosphorylated, subsequently leading to the nuclear translocation of the p65 to bind NF-KB-responsive genes(Mattson and Camandola, 2001). Inhibiting NF-KB activation is an effective strategy for reducing acute ischaemic stroke induced brain injury and inflammation (Li, Dai et al., 2019; Liang, Lin et al., 2019; Tan, Li et al., 2019).

Currently, recombinant tissue plasminogen activator (r-tPA) remains the only FDA-approved pharmacological treatment for stroke patients. However, few patients could benefit from it due to the rigid therapeutic time window and severe side effects. Thus, complementary and/or alternative medicine is urgently needed to treat ischaemic stroke worldwide. Electroacupuncture (EA) has been identified as a general complementary therapy for post-stroke patients in China, as it is more readily controlled, standardized, and objectively measurable(Cai, Zhang et al., 2017; Chen, Zhao et al., 2020; Fan, Kuang et al., 2020; Zhang, Wang et al., 2020; Zhu, Chen et al., 2020). Consistent with other reports(Liu, Wang et al., 2016), we also demonstrated that applying EA during the acute phase of cerebral ischaemic injury exerted potent neuroprotective effects by depressing overactivation of NF-KB signalling pathway(Zhan, Qin et al., 2016; Jiang, Luo et al., 2017; Xu, Mu et al., 2018). However, the specific mechanism remains unclear, and further exploration is needed.

Ovarian tumor domain deubiquitinase with linear linkage specificity (OTULIN) is a newly discovered deubiquitinase that selectively hydrolyses linear ubiquitin chains assembled by the linear ubiquitin chain assembly complex (LUBAC), and is regarded as an essential endogenous negative regulator for controlling inflammatory responses(Elliott, Nielsen et al., 2014; Damgaard, Walker et al., 2016; Zhou, Yu et al., 2016; Damgaard, Elliott et al., 2019; Nabavi, Shahrooei et al., 2019). OTULIN is expressed in immune cells, such as T cells, B cells, and natural killer cells, and prominently in dendritic cells and macrophages; moreover, it has been proven to be critical in the NF-KB-dependent inflammatory signalling pathway(Damgaard, Walker et al., 2016). OTULIN was also reported to protect liver against cell death, inflammation, fibrosis, and cancer(Damgaard, Jolin et al., 2020; Verboom, Martens et al., 2020) and is involved in regulating autophagy initiation and autophagosomes(Chu, Kang et al., 2020). However, the role of OTULIN in CNS is poorly understood, and its role in cerebral ischaemia is limited to a paper we published previously(Xu, Qin et al., 2018). OTULIN was enriched in activated microglia, and OTULIN overexpression ameliorated microglia-mediated neuroinflammation by regulating NF-KB activation in focal cerebral ischaemia/reperfusion rats(Xu, Qin et al., 2018).

Our previous studies demonstrated that neuronal zinc finger protein A20 and cylindromatosis (CYLD), which are important deubiquitinases that negatively regulate the NF-KB pathway, were required for EA to alleviate excessive inflammatory reaction in acute ischaemic rats(Zhan, Qin et al., 2016; Jiang, Luo et al., 2017). As the deubiquitination activity of OTULIN is more critical than A20 and CYLD in terms of regulating NF-KB pathway(Keusekotten, Elliott et al., 2013; Aksentijevich and Zhou, 2017), we speculated that EA may also alleviate brain injury and glial activation neuroinflammation following cerebral ischaemia by regulating OTULIN. Therefore, this study aimed to characterize the spatiotemporal 
expression of OTULIN, the effect of EA on OTULIN expression, and the possible mechanism by which EA regulates OTULIN-mediated neuroprotection.

\section{Materials And Methods}

\section{Animals}

A total of 313 Sprague-Dawley (SD) rats (250-300 g) were purchased from the Experimental Animal Center of Chongqing Medical University. All rats were housed under a 12/12 h light and dark cycle condition and allowed free access to food and water.

\section{Establishment of the transient middle cerebral artery occlusion (tMCAO) model}

The model was performed according to a method described previously(Longa, Weinstein et al., 1989; Qin, Luo et al., 2013). Briefly, rats were initially anaesthetized with $3.5 \%$ chloral hydrate $(1 \mathrm{ml} / 100 \mathrm{~g})$ intraperitoneally. After a midline neck incision, the bifurcation of the right common carotid artery (CCA) was exposed, and a heparin-dampened nylon monofilament with a rounded tip was advanced to block the origin of the right middle cerebral artery (MCA). Two hours after MCA occlusion, rats were reanaesthetized, and the monofilament was gently withdrawn to restore blood flow. All the same surgical procedures except the insertion of a monofilament to the origin of the MCA were performed in the Sham group. Successful tMCAO and reperfusion was determined by a decrease in the regional cerebral blood flow to $20 \%$ and recovery to $>80 \%$ of the baseline monitored by a laser-Doppler flowmeter (PeriFlux 5000 , Perimed $A B$, Sweden). Throughout the operation, the body temperature was continuously maintained at $38 \pm 0.5^{\circ} \mathrm{C}$ by a thermostatically controlled infrared lamp (FHC, Bowdoinham, ME, USA). After rats recovered from anaesthesia, the Longa score(Longa, Weinstein et al., 1989) was used to assess neurological deficits. Rats scoring 2 and 3 were included as previously described(Xu, Mu et al., 2018).

\section{Electroacupuncture (EA) treatment}

EA treatment was performed as before(Qin, Luo et al., 2013; Xie, Gao et al., 2016). The acupoints "Baihui (GV 20)", "Hegu (LI 4)", and "Taichong (LR 3)" on the left side of the rats were chosen (as shown in Fig. 1A). "Baihui (GV 20)" is located at the intersection of the sagittal midline and the line connecting the two ears, "Hegu (LI 4)" is at the second metacarpal midpoint of the radial side, and "Taichong (LR 3)" is at the second toe tibial collateral at the rear of the phalanx. The needle was inserted into each acupoint at an angle of $15^{\circ}-45^{\circ}$ with a 0.5 inch depth and then connected with the EA instrument (Model no. 227033; Beijing Jinggong Ltd., China). The acupoints were stimulated with an intensity of $1 \mathrm{~mA}$ and a frequency of $2 / 20 \mathrm{~Hz}$ for $30 \mathrm{~min}$ as the stimulation parameters can induce visible muscle contraction. The initial EA treatment was conducted once the reperfusion was completed and performed once daily thereafter.

\section{Lentivirus construction and intracerebroventricular administration}


The lentivirus for silencing OTULIN (LV-shOTULIN, $1 \times 10^{9}$ transduction units [TU]/ml) and control lentivirus (LV-Scramble, $1 \times 109$ transduction units $[\mathrm{TU}] / \mathrm{ml}$ ) were constructed by GenePharma Corporation (Shanghai, China).

Seven days before tMCAO, SD rats received intracerebroventricular (i.c.v) injection of LV-shOTULIN or LVScramble as before (Xu, Qin et al., 2018). Briefly, rats were anaesthetized and fixed in a stereotaxic apparatus (Stoelting, USA). LV-shOTULIN or LV-Scramble was injected into the right i.c.v. (bregma coordinates: $1.3 \mathrm{~mm}$ lateral, $1.5 \mathrm{~mm}$ posterior, and $3.8 \mathrm{~mm}$ under the dural surface) by a $10-\mu \mathrm{l}$ Hamilton syringe (Hamilton Co., Reno, NV, USA) at a rate of $0.5 \mu \mathrm{l} / \mathrm{min}$, and the needle was left in place for $5 \mathrm{~min}$ to prevent backflow. The animals were carefully monitored until recovery from anaesthesia. To verify the effect of gene silencing on OTULIN expression, OTULIN mRNA and protein were detected by RT-qPCR and Western blotting.

\section{Neurobehavioural assessment}

Assessments of neurobehavioural deficits were performed at $72 \mathrm{~h}$ with the modified Neurological Severity Score (mNSS)(Chen, Li et al., 2001), rotarod test(Linden, Fassotte et al., 2014) and inclined board test(Zhang, Wei et al., 2006) with some modifications by an independent investigator blinded to the experiment.

mNSS is a composite test including motor (muscle status and abnormal movement), sensory (visual, tactile and proprioceptive), reflex (pinna, corneal and startle), and balance assessments. It is graded on a scale of $0-18$, and the higher the score is, the more severe the injury.

The rotarod test was used to evaluate motor coordination by an accelerating rotarod apparatus (YLS-4C, Shanghai Jinggong Industry Co., Ltd, China). Briefly, each rat was placed on the rotating rod, which accelerates from 4 to $40 \mathrm{rpm}$ per min and went through three trials with $10 \mathrm{~min}$ breaks. A trial ended if the rat fell from the rotating rod or clung to the rod without walking for two consecutive rotations, and the latency to end was recorded. Before IMCAO, all rats were trained to stay on the rotating rod at a speed of $4 \mathrm{rpm}$ three times per day for three days. The pre-tMCAO data were recorded as the internal control. The rotarod test results are presented as the percentage of the mean latency time post-tMCAO compared with that pre-tMCAO. The lower the score was, the more severe the injury.

An inclined board test was used to assess balance and coordination. Animals were placed on a board $(50 \times 30 \mathrm{~cm})$ covered by copper wire mesh $(0.2 \mathrm{~mm})$ and stabilized. The board was gradually inclined from the horizontal to the vertical plane. The holding angle at which the animal fell from the board was recorded. The test was repeated and measured three times with two min intervals. The scores were recorded according to the holding angle as follows: $0,>70^{\circ} ; 1,65-69^{\circ} ; 2,60-64.9^{\circ} ; 3,55-59.9^{\circ} ; 4,<55^{\circ}$. The higher the score was, the more severe the injury.

\section{TTC staining}


The cerebral infarct volume was detected by 2,3,5-triphenyltetrazolium chloride (TTC, Sigma-Aldrich, USA) staining as described previously (Xu, Mu et al., 2018). Rats were euthanized, and brains were quickly removed and frozen for $20 \mathrm{~min}$ at $-20^{\circ} \mathrm{C}$. Then, the brains were coronally sliced into five 2-mm thick sections, stained with $2 \%$ TTC at $37^{\circ} \mathrm{C}$ for $10 \mathrm{~min}$, and then fixed with $10 \%$ formaldehyde. The stained sections were photographed by camera (Canon IXUS, Canon Co., Japan) and analysed by Image-Pro Plus (version 6.0, Media Cybernetics Co., USA). The infarct volume was calculated using the following formula: percentage hemisphere lesion volume=[total infarct volume-(right hemisphere volume-left hemisphere volume)]/left hemisphere volumex $100 \%$.

\section{Cresyl violet staining}

Frozen coronal brain sections were used for cresyl violet staining to label the ischaemic penumbra of the cerebral cortex in rats as previously described(Marchese, Occhieppo et al., 2020). The brain sections were dehydrated through descending grades of ethanol $(95 \%, 90 \%, 80 \%, 70 \%$, and $50 \%$ for $5 \mathrm{~min}$ ) and rinsed with water. The sections were then immersed in $0.1 \%$ cresyl violet solution for $10 \mathrm{~min}$, rinsed quickly in water, dehydrated though ascending grades of ethanol, immersed 3 times for 2 min each in xylene, and then cover-slipped.

\section{Nissl staining}

The frozen coronal brain sections were dehydrated through descending grades of ethanol $(95 \%, 90 \%$, $80 \%, 70 \%$, and $50 \%$ for $5 \mathrm{~min}$ ), immersed in $0.1 \%$ cresyl violet solution for $10 \mathrm{~min}$, rinsed quickly in water, rehydrated though ascending grades of ethanol, immersed 3 times for 2 min each in xylene, and then cover-slipped. The sections were photographed by a digital camera.

\section{Western blot}

Rats were sacrificed, and the brain tissues in the ischaemic penumbra were rapidly dissected. The tissues were homogenized in RIPA lysis buffer (no. P0013B, Beyotime, Shanghai, China) supplemented with PMSF (Beyotime, Shanghai, China) and phosphatase inhibitors for the detection of phosphorylated proteins. After centrifugation, total protein was extracted from the supernatant. The nuclear and cytoplasmic proteins were extracted by a Nuclear and Cytoplasmic Protein Extraction Kit (no. AR0106, Boster, Beijing, China), and the protein concentrations were detected by a BAC kit (Beyotime, Shanghai, China). SDS-PAGE was used to separate proteins, and PVDF membranes (Millipore Co., USA) were used to transfer proteins. After blocking in $5 \%$ non-fat milk for $2 \mathrm{~h}$, the PVDF membranes were incubated with the following primary antibodies at $4^{\circ} \mathrm{C}$ : anti-OTULIN polyclonal rabbit antibody (no. 14127, Cell Signaling Technology, USA, 1:1000), anti-NF-kB p65 rabbit antibody (no. 8242, Cell Signaling Technology, USA, 1:1000), anti-IKBa rabbit antibody (no. 4812, Cell Signaling Technology, USA, 1:1000), anti-phospho-IKBa (Ser32) rabbit antibody (no. 2859, Cell Signaling Technology, USA, 1:500), and anti- $\beta$-actin rabbit antibody (no. 4970, Cell Signaling Technology, USA, 1:1000). The next day, the membranes were incubated with a specific horseradish peroxidase-conjugated secondary antibody for $1 \mathrm{~h}$ at $37^{\circ} \mathrm{C}$. A gel imaging instrument 
(Vilber Lourmat fusion FX 7 Spectra, France) and analysis software (FUSION-CAPT, France) were applied to scan and analyse the immunoblots.

\section{Real-time quantitative reverse transcription polymerase chain reaction (RT-qPCR) analysis}

Total tissue RNA in the ischaemic penumbra of the cerebral cortex was isolated by TRIzol (Takara Biotechnology, Japan). The mRNA was used as a template to synthesize cDNA by using the PrimeScript ${ }^{\mathrm{TM}}$ RT reagent kit with gDNA Eraser (TaKaRa) at $42^{\circ} \mathrm{C}$ for $2 \mathrm{~min}$. The synthesized cDNA was used for RTqPCR on an iQ5 Gradient Real-Time PCR detection system (Bio-Rad Co., USA) with SYBR Green (SYBR Premix Ex Taq $\left.{ }^{\mathrm{TM}} \mathrm{II}, \mathrm{TaKaRa}\right)$. The cycling conditions were as follows: $10 \mathrm{~min}$ at $95^{\circ} \mathrm{C}$ followed by 40 cycles of $5 \mathrm{~s}$ at $95^{\circ} \mathrm{C}$ and $30 \mathrm{~s}$ at $60^{\circ} \mathrm{C}$. The melting curve was used to analyse the gene specificity of OTULIN and housekeeping gene $\beta$-Actin. Relative quantification was carried out with the $2^{-\Delta \Delta C t}$ method. The expression of each targeted gene was normalized to the expression of $\beta$-actin in the same sample. All primer sequences used in the study were as follows: for OTULIN, forward primer:

TGTGGCTCCTGAAATGGATATTATG, reverse primer: CTCTGACAGGGATGTTATAGTGCCG; for $\beta$-Actin, forward primer: TGTCACCAACTGGGACGATA, reverse primer: GGGGTGTTGAAGGTCTCAAA.

\section{Immunofluorescence and TUNEL staining}

Anaesthetized rats were perfused transcardially with $0.9 \%$ saline and $4 \%$ formaldehyde, and the brains were removed, fixed in $4 \%$ paraformaldehyde for $24 \mathrm{~h}$, and dehydrated with $30 \%$ sucrose, $20 \%$ sucrose and $15 \%$ sucrose. The brains were cut into $10 \mu \mathrm{m}$ thick coronal sections, incubated with $1 \%$ Triton X-100 for $30 \mathrm{~min}$, and blocked with $5 \%$ bovine serum albumin (BOSTER Co., USA) for $1 \mathrm{~h}$ at $37^{\circ} \mathrm{C}$. Then, the sections were incubated overnight at $4^{\circ} \mathrm{C}$ with the following primary antibodies: anti-OTULIN rabbit antibody (bs-14689R, Bioss Co., Beijing, China, 1:50), anti-NeuN mouse antibody (MAB377, Millipore Co., Germany, 1:200), anti-lba-1 goat antibody (NB100-1028, Novus Co., USA, 1:200), anti-GFAP mouse antibody (A00213, BOSTER Co.), and anti-NF-KB p65 rabbit antibody p65 (\#8242, Cell Signaling Technology, USA). The next day, the slices were incubated with the following secondary antibodies at $37^{\circ} \mathrm{C}$ for $1 \mathrm{~h}$ : Alexa Fluor 594-conjugated goat anti-rabbit IgG (H+L; SA00006-4, Proteintech, 1:200), Alexa Fluor 594-conjugated goat anti-mouse IgG $(\mathrm{H}+\mathrm{L})$ (SA00006-3, Proteintech, 1:100), Alexa Fluor 488conjugated goat anti-mouse IgG (H+L; SA00006-1, Proteintech, 1:200), FITC-conjugated AffiniPure donkey anti-goat IgG (H+L; SA00003-3, Proteintech, 1:200), or 594-conjugated AffiniPure donkey anti-rabbit IgG $(H+L ;$ SA00006-8, Proteintech, 1:200). The nuclei were stained with Dapi (Sigma, USA, 1:200). All images were captured using an A1+R laser confocal microscope (Nikon, Tokyo, Japan). Apoptotic cells were detected by TUNEL assay according to the manufacturer's instructions (Roche, Basel, Switzerland). The sections were photographed using an A1+R laser confocal microscope (Nikon, Tokyo, Japan).

Iba-1 is a commonly used microglial marker. In this study, the morphology of reactive microglia in the ischaemic penumbra of the cerebral cortex was determined by a classic method(Ito, Tanaka et al., 2001; Sawano, Watanabe et al., 2015). Briefly, according to the length of branches, thickness of branches and cell body size, Iba- $1^{+}$cells were categorized into the following three forms: 1 ) ramified microglia, with a 
small soma, and very fine, long processes; 2) internal microglia, with a larger cell body and thicker processes than ramified microglia; and 3) ameboid microglia, characterized by a large cell body without processes.

The morphological analysis of $\mathrm{GFAP}^{+}$astrocytes was mainly completed through a method described previously (Ramirez-Sanchez, Pires et al., 2018). Briefly, GFAP ${ }^{+}$cells with clearly visible nuclei and soma were selected, and the length of the longest cellular processes (the distance of the nucleus to the tip of the extension process) was measured as an indicator to assess the degree of hypertrophy of astrocytes according to a method described previously.

\section{Immunohistochemistry}

Paraffin-embedded brain sections were deparaffinized in xylene and immersed in citrate buffer for

antigen retrieval by microwave for $20 \mathrm{~min}$. Then, the endogenous peroxides were blocked by $3 \% \mathrm{H}_{2} \mathrm{O}_{2}$ for $30 \mathrm{~min}$, and nonspecific antigens were blocked by $5 \%$ goat serum for $30 \mathrm{~min}$ at $37^{\circ} \mathrm{C}$. The sections were incubated at $4^{\circ} \mathrm{C}$ overnight with primary antibodies IKBa rabbit monoclonal antibody (ab32518, Abcam, USA, 1:100) and anti-NF-KB p65 rabbit monoclonal antibody (\#8242, CST, USA, 1:400). The next day, the sections were incubated with secondary horseradish peroxidase-conjugated goat anti-rabbit (Proteintech ${ }^{\text {TM }}, 1: 2000$ ) and stained with 3,3囚-diaminobenzidine (DAB) substrate. Images were acquired using an automatic microscope (Olympus, Tokyo, Japan)(Qin, Luo et al., 2013).

\section{Enzyme-linked immunosorbent assay (ELISA)}

The levels of tumor necrosis factor alpha (TNF- $a$ ), interleukin-1 beta (IL-1 $\beta$ ) and interleukin-6 (IL-6) in brain tissues from the ischaemic penumbra of the cerebral cortex were detected using ELISA kits (no. EK0526 96T, EK0393 and EK0412 96T, BOSTER Co.) according to the manufacturer's instructions. The whole experimental flowchart is shown in

\section{Statistical analysis}

The neurological scores were analysed using Kruskal-Wallis tests followed by post hoc Dunn's multiple comparison tests, and all other data were expressed as the mean \pm SEM. The expression of OTULIN mRNA and protein among the Sham, tMCAO and EA groups was compared using two-way ANOVA with Bonferroni post hoc tests. All other quantitative data were analysed using one-way ANOVA followed by Tukey's post hoc test for multiple comparisons. Statistical analysis was performed using SPSS 19.0. $\mathrm{P}<0.05$ was considered statistically significant.

\section{Results}

\section{EA enhanced OTULIN expression following focal cerebral ischaemia/reperfusion injury}

To detect the effect of EA on the time course of OTULIN expression, OTULIN mRNA and protein in the ischaemic penumbra of the cerebral cortex (Fig.2A) from $2 \mathrm{~h}$ to $7 \mathrm{~d}$ after reperfusion were measured by 
RT-qPCR and Western blot, respectively. Rats were randomly divided into the Sham, tMCAO and tMCAO+EA groups. Compared with those in the Sham group, OTULIN mRNA and protein in the TMCAO group decreased obviously as early as $2 \mathrm{~h}$ and then increased gradually with a peak at $48 \mathrm{~h}$. The concentrations of OTULIN mRNA and protein in the TMCAO+EA group were increased significantly compared with those in the tMCAO group at the indicated time points except at $2 \mathrm{~h}$ and $6 \mathrm{~h}$ (Fig. 2B-D).

Next, the immune response of OTULIN protein in the ischaemic penumbra of the cerebral cortex was determined by immunofluorescence at $48 \mathrm{~h}$. The number of OTULIN ${ }^{+}$cells in the TMCAO group increased obviously compared with that in the Sham group $(P<0.01)$, and the count in the tMCAO+EA group was further increased compared with that in the tMCAO group $(P<0.01)$. In addition, the OTULIN protein was mainly located in the cytoplasm, and little OTULIN was expressed in the nucleus in all groups. (Fig. 2E, F).

\section{Enhanced OTULIN was mainly expressed in microglia and neurons in focal cerebral ischaemia/reperfusion rats}

Next, we explored the main cellular localization of OTULIN protein in the ischaemic penumbra of the cerebral cortex. Rats were randomly divided into the Sham, tMCAO and tMCAO+EA groups. Forty-eight hours, the peak time for OTULIN expression, was selected as the observation time point. OTULIN was costained with NeuN, Iba-1 and GFAP by immunofluorescence staining.

As shown in Fig. 3, in the Sham group, OTULIN was mainly located in $\mathrm{NeuN}^{+}$cells, and little OTULIN was detected in Iba- $1^{+}$cells. OTULIN was predominantly expressed in Iba- $1^{+}$cells, and little OTULIN was observed in reduced $\mathrm{NeuN}^{+}$cells in the tMCAO group. Moreover, OTULIN protein in the TMACO+EA group was upregulated in both $\mathrm{NeuN}^{+}$and $\mathrm{Iba}-1^{+}$cells compared with that in the TMCAO group. No OTULIN expression was observed in $\mathrm{GFAP}^{+}$cells in any group.

\section{OTULIN knockdown reversed EA's neuroprotective effect against ischaemic brain injury}

To explore whether enhanced OTULIN expression was required for EA to exert a neuroprotective effect, gene interference by LV-shOTULIN was applied to silence OTULIN expression. Rats were randomly divided into five groups: Sham, tMCAO, tMCAO+EA, tMCAO+EA+LV-Scramble, and tMCAO+EA+LV-shOTULIN. Seventy-two hours after reperfusion, the levels of OTULIN mRNA and protein in the TMCAO+EA+LVshOTULIN group were significantly decreased compared with those in the TMCAO+EA and TMCAO+EA+LVscramble groups (Fig. 4A-C).

The neurobehavioural assessment with the mNSS, inclined board test and rotarod test, and cerebral infarct volume detection were performed at $72 \mathrm{~h}$. As shown in Fig. 4D-H, rats in the tMCAO group showed more severe neurobehavioural dysfunction than rats in the Sham group. EA obviously improved neurological function, as demonstrated by a lower mNSS score, higher holding angle and relative latency time than those in the TMCAO group. However, this improvement was weakened by LV-shOTULIN, as evidenced by a poor neurobehavioural improvement in the tMCAO+EA+LV-shOTULIN group. Moreover, as shown in Fig. 4G and H, no detectable cerebral infarct volume was found in the Sham group (Fig. 4G, H). 
The infarct volume in the $\mathrm{MCAO}+\mathrm{EA}$ group was decreased compared with that in the $\mathrm{MCAO}$ group. After silencing OTULIN, EA failed to reduce the infarct volume.

\section{Enhanced OTULIN expression was necessary for EA to attenuate neuronal injury in focal cerebral ischaemia/reperfusion rats}

Since OTULIN was also detected in neurons, we sought to investigate the effects of OTULIN on EA's role in attenuating neuronal injury in the ischaemic penumbra of the cerebral cortex at $24 \mathrm{~h}$. Rats were randomly divided into the following five groups: Sham, tMCAO, tMCAO+EA, tMCAO+EA+LV-Scramble and tMCAO+EA+LV-shOTULIN groups. First, viable neurons were detected by Nissl staining. As shown in Fig.

$5 A$ and $B$, neurons were arranged regularly, and most cells exhibited a normal shape in the Sham group. In the tMCAO group, most neurons were shrunken, deeply stained and sparsely distributed with a cytosolic concentration, karyopyknosis and karyorrhexis. In the tMCAO+EA group, the morphology of neurons was improved, with many normal-shaped cells, and the number of viable neurons was increased compared with that in the tMCAO group $(P<0.01)$. However, this improvement was obviously blocked by OTULIN silencing $(P<0.01)$. In Fig. $5 \mathrm{C}-\mathrm{E}$, a large number of $\mathrm{TUNEL}^{+}$and $\mathrm{TUNEL}^{+} \mathrm{NeuN}^{+}$cells were observed in the tMCAO group, whereas few apoptotic cells were detected in the Sham group. EA treatment significantly decreased the proportion of TUNEL ${ }^{+}$and $\mathrm{TUNEL}^{+} \mathrm{NeuN}^{+}$cells compared with $\mathrm{TMCAO}$ $(P<0.001)$, but the effect was inhibited by OTULIN silencing.

Next, we explored whether OTULIN participated in EA regulation of neuronal NF-KB p65 nuclear translocation. As shown in Fig. 5F, neuronal NF-KB p65 was mainly expressed in the nucleus in the tMCAO group. An increased concentration of NF-KB p65 protein was translocated to neuronal cytoplasm in the tMCAO+EA group compared with the TMCAO group, but this effect was reversed by OTULIN silencing.

\section{Increased OTULIN expression was required for EA to inhibit the NF-KB signalling pathway in focal cerebral ischaemia/reperfusion rats}

To further investigate whether enhanced OTULIN was required for EA to inhibit the NF-KB signalling pathway, OTULIN, p-IKBa, IKBa, cytoplasm-p65 and nucleus-p65 proteins from brain tissues in the ischaemic penumbra of the cerebral cortex were detected $24 \mathrm{~h}$ after reperfusion. $\mathrm{p}-\mathrm{I} \mathrm{kBa} / \mathrm{I} \mathrm{kBa}$ represents the phosphorylation ratio of $\mathrm{IKBa}$, and nuclear/cytoplasmic NF-KB p65 represents the nuclear translocation of NF-KB p65 protein. Rats were randomly divided into the tMCAO, TMCAO+EA, tMCAO+EA+LV-Scramble and tMCAO+EA+LV-shOTULIN groups. As shown in Fig. 6A and B, increased OTULIN protein content, a lower phosphorylation ratio of $\mathrm{IKBa}$, and attenuated nuclear translocation of p65 protein were detected in the TMCAO+EA group compared with the TMCAO group. In the MCAO+EA+LVshOTULIN group, little OTULIN was detected, and an elevated phosphorylation ratio of IKBa and increased nuclear translocation of NF-KB p65 were detected compared with those in the tMCAO+EA and tMCAO+EA+LV-Scramble groups. 
In addition, immunohistochemical analysis was also applied to detect IкBa and NF-KB p65 proteins $24 \mathrm{~h}$ after reperfusion. As shown in Fig. 6C, EA obviously enhanced ІкBa staining, and IкBa staining was obviously attenuated in the $\mathrm{MCAO}+\mathrm{EA}+\mathrm{LV}$-shOTULIN group compared with the tMCAO+EA group. NF-KB p65 protein was predominantly located in the nucleus in the IMCAO group but partly transferred to the cytoplasm in the tMCAO+EA group. In the tMCAO+EA+LV-shOTULIN group, NF-KB p65 staining increased in the nucleus and decreased obviously in the cytoplasm compared with the tMCAO+EA and tMCAO+EA+LV-Scramble groups.

\section{OTULIN silencing reversed the depression of microglial activation by EA after focal cerebral ischaemia/reperfusion injury}

The transformation of microglial shape is a manifestation of microglia activation. Microglia located in the ischaemic penumbra of the cerebral cortex were mainly in the following three forms: ramified cells (Fig. 7Aa), internal cells (Fig. 7Ab) and ameboid cells (Fig. 7Ac). To investigate the effect of OTULIN on EA's role in attenuating microglial activation, mean Iba-1 immunofluorescence intensity and the proportions of microglia with different forms were analysed quantitatively. All rats were randomly divided into the following five groups: Sham, $\mathrm{TMCAO}$, $M C A O+E A, \mathrm{M} C A O+E A+L V-S c r a m b l e ~ a n d ~ M C A O+E A+L V-$ shOTULIN groups.

First, the Iba-1 mean immunofluorescence intensity in each group was analysed. Twenty-four hours and $72 \mathrm{~h}$ after reperfusion, as shown in Fig. 7, the mean Iba-1 immunofluorescence intensity was very low in the Sham group and increased sharply in the tMCAO group, but it was obviously reduced in the tMCAO+EA group. After silencing OTULIN, the Iba-1 mean immunofluorescence intensity in the TMCAO+EA+LV-shOTULIN group was increased significantly compared with that in the TMCAO+EA and tMCAO+EA+LV-Scramble groups.

Next, the effect of EA and OTULIN silencing on the cell shape of microglia in the ischaemic penumbra was analysed. As shown in Fig. 7B, D, the main type of microglia was internal shape in the TMCAO group at $24 \mathrm{~h}$. In the tMCAO+EA group, the proportion of internal microglia was decreased, and the proportion of ramified microglia was obviously increased compared with the TMCAO group. After silencing OTULIN, the proportion of ramified microglia was significantly lower, while the proportion of internal microglia was significantly higher in the TMCAO+EA+LV-shOTULIN group than those in the TMCAO+EA and tMCAO+EA+LV-Scramble groups. At $72 \mathrm{~h}$, activated microglia were mainly in ameboid form (Fig. 7E, G). In the $\mathrm{TMCAO}+\mathrm{EA}$ group, the proportion of internal microglia was increased, and the proportion of ameboid microglia was obviously decreased compared with those in the tMCAO group. In the TMCAO+EA+LVshOTULIN group, the proportion of internal microglia was obviously decreased, and the proportion of ramified microglia was higher than those in the tMCAO+EA and tMCAO+EA+LV-Scramble groups.

To investigate the effect of OTULIN silencing on EA's role in depressing the secretion of pro-inflammatory cytokines, the contents of TNF- $\alpha$, IL-1 $\beta$ and IL- 6 were detected at $24 \mathrm{~h}$ and $72 \mathrm{~h}$ by ELISA. As shown in Fig. $7 \mathrm{H}-\mathrm{J}$, the levels of TNF-a, IL-1 $\beta$, and IL- 6 in the tMCAO group were significantly higher than those in the Sham group, and they decreased after EA treatment. After silencing OTULIN expression, the contents of 
TNF-a, IL-1 $\beta$ and IL-6 in the tMCAO+EA+LV-shOTULIN group were significantly higher than those in the tMCAO+EA and tMCAO+EA+LV-Scramble groups.

\section{OTULIN reversed the depression of astrogliosis by EA in focal cerebral ischaemia/reperfusion rats}

Although OTULIN protein was not detected in astrocytes, we tried to explore whether the attenuated neuroinflammation could adversely affect the activation of astrocytes. Rats were randomly divided into the Sham, tMCAO, tMCAO+EA, tMCAO+EA+LV-Scramble and tMCAO+EA+LV-shOTULIN groups. Immunofluorescence was used to observe $\mathrm{GFAP}^{+}$astrocytes in the ischaemic penumbra of the cerebral cortex at $24 \mathrm{~h}$ and $72 \mathrm{~h}$. The mean immunofluorescence intensity of GFAP and the mean longest process of $\mathrm{GFAP}^{+}$cells were quantitatively analysed.

In the tMCAO group, the mean immunofluorescence intensity of GFAP and the mean longest process of $\mathrm{GFAP}^{+}$cells were increased significantly compared with the Sham and EA group at both $24 \mathrm{~h}$ and $72 \mathrm{~h}$. After silencing OTULIN, the mean immunofluorescence intensity of GFAP in the tMCAO+EA+LV-shOTULIN group was significantly higher than that in the tMCAO+EA group at $72 \mathrm{~h}$, but not at $24 \mathrm{~h}$, while the mean longest process of $\mathrm{GFAP}^{+}$cells was increased significantly compared with that in the tMCAO+EA group and tMCAO+EA+LV-Scramble group at both $24 \mathrm{~h}$ and $72 \mathrm{~h}$.

\section{Discussion}

OTULIN, a crucial negative regulator of the NF-KB signalling pathway, has been proven to be critical for preventing autoinflammatory diseases(Damgaard, Walker et al., 2016; Zhou, Yu et al., 2016; Damgaard, Jolin et al., 2020) and protecting embryonic lethality(Rivkin, Almeida et al., 2013). Genetic mutation of OTULIN in humans causes a severe inflammatory phenotype, and patients exhibit amplified activation of the NF-KB pathway (Damgaard, Walker et al., 2016). Mice that lacked OTULIN in myeloid cells exhibited enlarged lymphoid organs and liver, increased immune cell infiltration in liver, and increased cytokine levels in serum(Damgaard, Walker et al., 2016). We previously demonstrated that OTULIN overexpression ameliorated microglial activation and neuroinflammation by depressing the NF-kB pathway in cerebral ischaemia/reperfusion rats(Xu, Qin et al., 2018). In this study, rapidly decreased OTULIN was detected as early as $2 \mathrm{~h}$ following perfusion. It is speculated that hyperacute brain injury inhibits brain cells from synthesizing OTULIN. Subsequently, endogenous OTULIN expression increased gradually with a peak at $48 \mathrm{~h}$, which is considered an endogenous protection, as we previously demonstrated that enhanced OTULIN exerted a neuroprotective role in ischaemic stroke.

In this study, for the first time, OTULIN was detected to be located mainly in neurons and microglia in both rats in a normal physiological state and those with ischaemic stroke, but no OTULIN protein was detected in astrocytes. EA treatment could promote OTULIN gene transcription and protein synthesis in the brain, and enhanced OTULIN was mainly located expressed in neurons. Importantly, the neuroprotective role of EA was obviously reversed by silencing OTULIN, which indicated that OTULIN was involved in the antipreventive role of EA following acute ischemic stroke. 
NF-KB signalling pathway is widely activated in brain cells after cerebral ischaemia, while its effect on neuronal injury is controversial. Some studies reported that neuronal conditional NF-KB knockout mice exhibited impaired synaptic transmission, spatial memory formation, and plasticity(O'Mahony, Raber et al., 2006), while other studies showed that NF-KB activation in neurons contributed to neuronal cell death in cerebral ischaemia(Zhang, Potrovita et al., 2005; Ridder and Schwaninger, 2009; Ingrassia, Lanzillotta et al., 2012). Sarnico et al(Sarnico, Lanzillotta et al., 2009) found that overexpression of p65 reduced neuron susceptibility to anoxia, and p65 silencing significantly reduced neuronal cell death in vitro. Although the functional consequences of neuronal NF-KB and neuronal survival are still disputed, EA has been confirmed to decrease neuronal apoptosis and improve neurological function by inhibiting NF-KB activation in the whole brain. In this study, it was shown that alleviated neuronal injury and apoptosis by EA was accompanied by enhanced OTULIN expression and depressed activation of the neuronal NF-KB signalling pathway, while the above findings were obviously reversed when OTULIN expression was inhibited. However, whether enhanced neuronal OTULIN is directly required for attenuating neuronal injury by EA is unclear and needs further exploration, as this effect may partly be attributed to alleviated neuroinflammation.

EA treatment reduces microglial activation after cerebral ischaemia/reperfusion injury, and the degree of microglial activation is often assessed by calculating the number of Iba- $1^{+}$cells or mean Iba- 1 immunofluorescence intensity(Han, Lu et al., 2015; Liu, Wang et al., 2016; Zhan, Qin et al., 2016; Jiang, Luo et al., 2017; Xu, Mu et al., 2018). In this study, EA could also enhance microglial OTULIN expression, and OTULIN silencing effectively reversed the inhibitory effect of EA on microglial activation, as shown by increased mean Iba-1 immunofluorescence intensity, which strongly indicates that enhanced OTULIN expression is necessary for EA to alleviate microglial activation.

Morphological changes in microglia are a reflection of microglial activation(Soltys, Orzylowska-Sliwinska et al., 2005; Heindl, Gesierich et al., 2018; Li, Lang et al., 2018). However, little was known about EA's effect on microglial morphology following cerebral ischaemia until now. By quantitative analysis of microglia with different shapes using immunofluorescence, this study revealed that EA inhibited the transformation of microglia from the ramified to internal state at $24 \mathrm{~h}$ and transformation of microglia from the internal to ameboid shape at $72 \mathrm{~h}$. Importantly, these transformations were partly inhibited by OTULIN silencing, which suggests that OTULIN is involved in EA's role in inhibiting the transformation of microglia from resting state to active state. In line with our hypothesis, OTULIN silencing reversed the inhibitory effect of EA on the secretion of pro-inflammatory factors in the ischaemic cerebral cortex. EA effectively inhibits the activation of microglia and the secretion of inflammatory mediators by regulating OTULIN expression.

Astrocytes, the most abundant cells in the CNS, change to a reactive phenotype (so-called reactive astrogliosis) characterized by enhanced glial fibrillary acidic protein (GFAP) expression and cellular hypertrophy(Sofroniew, 2009; Pekny and Pekna, 2014; Rossi, 2015) in response to ischaemic stroke. Numerous studies have proven that EA can inhibit astrocyte activation induced by acute cerebral ischaemia/reperfusion injury(Tian, Tao et al., 2016; Zhan, Qin et al., 2016; Kim, Koo et al., 2018), 
traumatic brain injury(Tang, Hsu et al., 2016), neuropathic pain(Liang, Qiu et al., 2016), and inflammatory pain(Liao, Hsieh et al., 2017). Although astrogliosis plays a dual role in ischaemic stroke, inhibition of excessive astrogliosis was considered beneficial(Zhang, Wu et al., 2018), as reactive astrogliosis and glial scar formation are the main reasons for functional recovery difficulty after cerebral ischaemia(Cregg, DePaul et al., 2014; Cheon, Cho et al., 2016; Liu and Chopp, 2016). It is well demonstrated that EA could inhibit astrogliosis(Tian, Tao et al., 2016; Zhan, Qin et al., 2016), and this is beneficial to the antiinflammatory effect of EA(Zhan, Qin et al., 2016). Microglia and astrocytes are the primary immune cell types that quickly respond to cerebral ischaemia, but their functions are increasingly recognized as being complex and interactive (sometimes even synergistic)(Liddelow, Guttenplan et al., 2017; Zhang, Zhao et al., 2018). Actually, the complex cell-cell interactions have hampered the mechanistic understanding of astrocyte reactivity. As OTULIN was required for EA to alleviate microglial activation and neuroinflammation, we wondered whether astrogliosis could be affected after silencing OTULIN. Although no OTULIN was detected to be expressed in astrocytes, EA failed to depress astrocyte activation after silencing OTULIN. It was speculated to be related to attenuated microglia activation, as it was reported that reactive microglia were required to induce reactive astrocytes by secreting various inflammatory factors, such as TNF-a, IL-1a, and complement component 1q (C1q)(Liddelow, Guttenplan et al., 2017). However, the specific mechanism needs deeper study.

However, there are some limitations in this study. OTULIN is a new type of deubiquitinating enzyme. After EA up-regulated the expression of OTULIN, how does OTULIN inhibited the activation of NF-KB signaling pathway? IKKY is an important modification target of OTULIN in the NF-KB signaling pathway, and how does its ubiquitination modification level change? Which will be demonstrated in later studies.

\section{Conclusion}

Our data highlight the temporal and spatial distribution of OTULIN in the cerebral cortex following ischaemic stroke and revealed that enhanced OTULIN is required for EA to alleviate neuronal injury and the activation of glial cells, which was associated with NF-KB signalling pathway. Although there are still many problems that need to be solved, this study proposes OTULIN as a new target that exerts a neuroprotective role in ischaemic stroke, and this may be a complex process in which a variety of cells participate and coordinate with each other.

\section{Abbreviations}

tMCAO: transient Middle cerebral artery occlusion; OTULIN: Ovarian tumor domain deubiquitinase with linear linkage specificity; EA: Electroacupuncture; CCA: common carotid artery; MCA: middle cerebral artery; CNS: Central nervous system; i.c.v: intracerebroventricular; TTC: 2,3,5-Triphenyltetrazolium chloride; Iba-1: Ionized calcium binding adapter molecule 1; IkBa: NF-кB inhibitor protein alpha; NF-kB: Nuclear transcription factor kappaB; TNF-a: Tumor necrosis factor alpha; IL-1 B: Interleukin-1 beta; IL-6: Interleukin6; RT-qPCR: Real-time quantitative polymerase chain reaction; ELISA: Enzyme-linked immunosorbent assay. 


\section{Declarations}

Acknowledgements

Not applicable

\section{Funding}

This study was supported by Youth Program of National Natural Science Foundation of China (Grant No. 82001270), and the Chinese Medicine Science and Technology Project of Chongqing Municipal Health Bureau Grant (20a2-2-a28).

\section{Authors' contributions}

HX designed the study, collected and analyzed the data, and drafted the manuscript. YL handled the funding, provided useful advice on the design of this study, supervised experimental work and revision of this manuscript. YW performed the experiments.

\section{Availability and materials}

The datasets used or analyzed during the current study are available from the corresponding author on reasonable request.

\section{Ethics approval and consent to participate}

The experimental protocol was approved by the Ethics Committee for Animal Experimentation of the First Affiliated Hospital of Chongqing Medical University. All procedures carried out were in strict compliance with the guidelines of the National Institutes for Animal Research.

\section{Consent for publication}

Not applicable

\section{Competing interests}

The authors declare that they have no competing interests.

\section{Author details}

${ }^{1}$ Department of Neurology, the Affiliated Hospital of Guizhou Medical University, 550004 Guizhou, China.

2 Department of Neurology, the First Affiliated Hospital of Chongqing Medical University, 400016 Chongqing, China. ${ }^{3}$ Laboratory Research Center, the First Affiliated Hospital of Chongqing Medical University, 400016 Chongqing, China. ${ }^{4}$ Department of Neurology, the Second Affiliated Hospital of Chongqing Medical University, 400016 Chongqing, China. 


\section{References}

1. Aksentijevich I and Zhou Q. NF-kappaB Pathway in Autoinflammatory Diseases: Dysregulation of Protein Modifications by Ubiquitin Defines a New Category of Autoinflammatory Diseases. Frontiers in immunology 2017;8:399

2. Cai Y, Zhang CS, Liu S, Wen Z, Zhang AL, Guo X, Lu C and Xue CC. Electroacupuncture for Poststroke Spasticity: A Systematic Review and Meta-Analysis. Arch Phys Med Rehabil 2017;98:2578-2589 e4

3. Chen B, Zhao M, Chen B, Yang Z, Yu X, Lin X and Fan C. Effectiveness and safety of acupuncture in post-stroke depression (PSD): Protocol for a Bayesian analysis. Medicine 2020;99:e18969

4. Chen J, Li Y, Wang L, Zhang Z, Lu D, Lu M and Chopp M. Therapeutic benefit of intravenous administration of bone marrow stromal cells after cerebral ischemia in rats. Stroke 2001;32:1005-11

5. Cheon SY, Cho KJ, Song J and Kim GW. Knockdown of apoptosis signal-regulating kinase 1 affects ischaemia-induced astrocyte activation and glial scar formation. Eur J Neurosci 2016;43:912-22

6. Chu Y, Kang Y, Yan C, Yang C, Zhang T, Huo H and Liu Y. LUBAC and OTULIN regulate autophagy initiation and maturation by mediating the linear ubiquitination and the stabilization of ATG13. Autophagy 2020

7. Corps KN, Roth TL and McGavern DB. Inflammation and neuroprotection in traumatic brain injury. JAMA neurology 2015;72:355-62

8. Cregg JM, DePaul MA, Filous AR, Lang BT, Tran A and Silver J. Functional regeneration beyond the glial scar. Exp Neurol 2014;253:197-207

9. Damgaard RB, Elliott PR, Swatek KN, Maher ER, Stepensky P, Elpeleg O, Komander D and Berkun Y. OTULIN deficiency in ORAS causes cell type-specific LUBAC degradation, dysregulated TNF signalling and cell death. EMBO molecular medicine 2019;11

10. Damgaard RB, Jolin HE, Allison MED, Davies SE, Titheradge HL, McKenzie ANJ and Komander D. OTULIN protects the liver against cell death, inflammation, fibrosis, and cancer. Cell death and differentiation 2020;27:1457-1474

11. Damgaard RB, Walker JA, Marco-Casanova P, Morgan NV, Titheradge HL, Elliott PR, McHale D, Maher ER, McKenzie ANJ and Komander D. The Deubiquitinase OTULIN Is an Essential Negative Regulator of Inflammation and Autoimmunity. Cell 2016;166:1215-1230 e20

12. Elliott PR, Nielsen SV, Marco-Casanova P, Fiil BK, Keusekotten K, Mailand N, Freund SM, Gyrd-Hansen $\mathrm{M}$ and Komander D. Molecular basis and regulation of OTULIN-LUBAC interaction. Molecular cell 2014;54:335-48

13. Fan W, Kuang X, Hu J, Chen X, Yi W, Lu L, Xu N and Wang L. Acupuncture therapy for poststroke spastic hemiplegia: A systematic review and meta-analysis of randomized controlled trials. Complementary therapies in clinical practice 2020;40:101176

14. Han B, Lu Y, Zhao H, Wang Y, Li L and Wang T. Electroacupuncture modulated the inflammatory reaction in MCAO rats via inhibiting the TLR4/NF-kappaB signaling pathway in microglia. Int $\mathrm{J}$ Clin Exp Pathol 2015;8:11199-205 
15. Heindl S, Gesierich B, Benakis C, Llovera G, Duering M and Liesz A. Automated Morphological Analysis of Microglia After Stroke. Front Cell Neurosci 2018;12:106

16. Ingrassia R, Lanzillotta A, Sarnico I, Benarese M, Blasi F, Borgese L, Bilo F, Depero L, Chiarugi A, Spano PF and Pizzi M. 1B/(-)IRE DMT1 expression during brain ischemia contributes to cell death mediated by NF-kappaB/RelA acetylation at Lys310. PLoS One 2012;7:e38019

17. Ito D, Tanaka K, Suzuki S, Dembo T and Fukuuchi Y. Enhanced expression of Iba1, ionized calciumbinding adapter molecule 1, after transient focal cerebral ischemia in rat brain. Stroke 2001;32:120815

18. Jiang J, Luo Y, Qin W, Ma H, Li Q, Zhan J and Zhang Y. Electroacupuncture Suppresses the NFkappaB Signaling Pathway by Upregulating Cylindromatosis to Alleviate Inflammatory Injury in Cerebral Ischemia/Reperfusion Rats. Frontiers in molecular neuroscience 2017;10:363

19. Kaushal V and Schlichter LC. Mechanisms of microglia-mediated neurotoxicity in a new model of the stroke penumbra. J Neurosci 2008;28:2221-30

20. Keusekotten K, Elliott PR, Glockner L, Fiil BK, Damgaard RB, Kulathu Y, Wauer T, Hospenthal MK, GyrdHansen M, Krappmann D, Hofmann K and Komander D. OTULIN antagonizes LUBAC signaling by specifically hydrolyzing Met1-linked polyubiquitin. Cell 2013;153:1312-26

21. Kim H, Koo YS, Shin MJ, Kim SY, Shin YB, Choi BT, Yun YJ, Lee SY and Shin HK. Combination of Constraint-Induced Movement Therapy with Electroacupuncture Improves Functional Recovery following Neonatal Hypoxic-Ischemic Brain Injury in Rats. BioMed research international 2018;2018:8638294

22. Li D, Lang W, Zhou C, Wu C, Zhang F, Liu Q, Yang S and Hao J. Upregulation of Microglial ZEB1 Ameliorates Brain Damage after Acute Ischemic Stroke. Cell Rep 2018;22:3574-3586

23. Li Q, Dai Z, Cao Y and Wang L. Caspase-1 inhibition mediates neuroprotection in experimental stroke by polarizing M2 microglia/macrophage and suppressing NF-kappaB activation. Biochemical and biophysical research communications 2019;513:479-485

24. Liang W, Lin C, Yuan L, Chen L, Guo P, Li P, Wang W and Zhang X. Preactivation of Notch1 in remote ischemic preconditioning reduces cerebral ischemia-reperfusion injury through crosstalk with the NFkappaB pathway. Journal of neuroinflammation 2019;16:181

25. Liang Y, Qiu Y, Du J, Liu J, Fang J, Zhu J and Fang J. Inhibition of spinal microglia and astrocytes contributes to the anti-allodynic effect of electroacupuncture in neuropathic pain induced by spinal nerve ligation. Acupunct Med 2016;34:40-7

26. Liao HY, Hsieh CL, Huang CP and Lin YW. Electroacupuncture Attenuates Induction of Inflammatory Pain by Regulating Opioid and Adenosine Pathways in Mice. Sci Rep 2017;7:15679

27. Liddelow SA, Guttenplan KA, Clarke LE, Bennett FC, Bohlen CJ, Schirmer L, Bennett ML, Munch AE, Chung WS, Peterson TC, Wilton DK, Frouin A, Napier BA, Panicker N, Kumar M, Buckwalter MS, Rowitch DH, Dawson VL, Dawson TM, Stevens B and Barres BA. Neurotoxic reactive astrocytes are induced by activated microglia. Nature 2017;541:481-487 
28. Lin SY, Wang YY, Chang CY, Wu CC, Chen WY, Kuan YH, Liao SL and Chen CJ. Effects of betaAdrenergic Blockade on Metabolic and Inflammatory Responses in a Rat Model of Ischemic Stroke. Cells 2020;9

29. Linden J, Fassotte L, Tirelli E, Plumier JC and Ferrara A. Assessment of behavioral flexibility after middle cerebral artery occlusion in mice. Behav Brain Res 2014;258:127-37

30. Liu JQ, Zhao M, Zhang Z, Cui LY, Zhou X, Zhang W, Chu SF, Zhang DY and Chen NH. Rg1 improves LPS-induced Parkinsonian symptoms in mice via inhibition of NF-kappaB signaling and modulation of M1/M2 polarization. Acta pharmacologica Sinica 2020;41:523-534

31. Liu W, Wang X, Yang S, Huang J, Xue X, Zheng Y, Shang G, Tao J and Chen L. Electroacupunctre improves motor impairment via inhibition of microglia-mediated neuroinflammation in the sensorimotor cortex after ischemic stroke. Life sciences 2016;151:313-322

32. Liu X, Zhang $X$, Wang F, Liang $X$, Zeng Z, Zhao J, Zheng H, Jiang $X$ and Zhang Y. Improvement in cerebral ischemia-reperfusion injury through the TLR4/NF-kappaB pathway after Kudiezi injection in rats. Life sciences 2017;191:132-140

33. Liu Z and Chopp M. Astrocytes, therapeutic targets for neuroprotection and neurorestoration in ischemic stroke. Prog Neurobiol 2016;144:103-20

34. Longa EZ, Weinstein PR, Carlson S and Cummins R. Reversible middle cerebral artery occlusion without craniectomy in rats. Stroke 1989;20:84-91

35. Marchese NA, Occhieppo VB, Basmadjian OM, Casarsa BS, Baiardi G and Bregonzio C. Angiotensin II modulates amphetamine-induced glial and brain vascular responses, and attention deficit via angiotensin type 1 receptor: Evidence from brain regional sensitivity to amphetamine. The European journal of neuroscience 2020;51:1026-1041

36. Mattson MP and Camandola S. NF-kappaB in neuronal plasticity and neurodegenerative disorders. J Clin Invest 2001;107:247-54

37. Nabavi M, Shahrooei M, Rokni-Zadeh H, Vrancken J, Changi-Ashtiani M, Darabi K, Manian M, Seif F, Meyts I, Voet A, Moens $L$ and Bossuyt X. Auto-inflammation in a Patient with a Novel Homozygous OTULIN Mutation. Journal of clinical immunology 2019;39:138-141

38. O'Mahony A, Raber J, Montano M, Foehr E, Han V, Lu SM, Kwon H, LeFevour A, Chakraborty-Sett S and Greene WC. NF-kappaB/Rel regulates inhibitory and excitatory neuronal function and synaptic plasticity. Mol Cell Biol 2006;26:7283-98

39. Pekny M and Pekna M. Astrocyte reactivity and reactive astrogliosis: costs and benefits. Physiol Rev 2014;94:1077-98

40. Qin WY, Luo Y, Chen L, Tao T, Li Y, Cai YL and Li YH. Electroacupuncture Could Regulate the NFkappaB Signaling Pathway to Ameliorate the Inflammatory Injury in Focal Cerebral Ischemia/Reperfusion Model Rats. Evid Based Complement Alternat Med 2013;2013:924541

41. Ramirez-Sanchez J, Pires ENS, Meneghetti A, Hansel G, Nunez-Figueredo Y, Pardo-Andreu GL, OchoaRodriguez E, Verdecia-Reyes Y, Delgado-Hernandez R, Salbego C and Souza DO. JM-20 Treatment 
After MCAO Reduced Astrocyte Reactivity and Neuronal Death on Peri-infarct Regions of the Rat Brain. Mol Neurobiol 2018

42. Ridder DA and Schwaninger M. NF-kappaB signaling in cerebral ischemia. Neuroscience 2009;158:995-1006

43. Rivkin E, Almeida SM, Ceccarelli DF, Juang YC, MacLean TA, Srikumar T, Huang H, Dunham WH, Fukumura R, Xie G, Gondo Y, Raught B, Gingras AC, Sicheri F and Cordes SP. The linear ubiquitinspecific deubiquitinase gumby regulates angiogenesis. Nature 2013;498:318-24

44. Rossi D. Astrocyte physiopathology: At the crossroads of intercellular networking, inflammation and cell death. Prog Neurobiol 2015;130:86-120

45. Sarnico I, Lanzillotta A, Benarese M, Alghisi M, Baiguera C, Battistin L, Spano P and Pizzi M. NFkappaB dimers in the regulation of neuronal survival. Int Rev Neurobiol 2009;85:351-62

46. Sawano T, Watanabe F, Ishiguchi M, Doe N, Furuyama T and Inagaki S. Effect of Sema4D on microglial function in middle cerebral artery occlusion mice. Glia 2015;63:2249-59

47. Shan HM, Zang M, Zhang Q, Shi RB, Shi XJ, Mamtilahun M, Liu C, Luo LL, Tian X, Zhang Z, Yang GY, Tang $Y, P u J$ and Wang $Y$. Farnesoid $X$ receptor knockout protects brain against ischemic injury through reducing neuronal apoptosis in mice. Journal of neuroinflammation 2020;17:164

48. Sofroniew MV. Molecular dissection of reactive astrogliosis and glial scar formation. Trends Neurosci 2009;32:638-47

49. Soltys Z, Orzylowska-Sliwinska O, Zaremba M, Orlowski D, Piechota M, Fiedorowicz A, Janeczko K and Oderfeld-Nowak B. Quantitative morphological study of microglial cells in the ischemic rat brain using principal component analysis. J Neurosci Methods 2005;146:50-60

50. Tan L, Li J, Wang Y and Tan R. Anti-Neuroinflammatory Effect of Alantolactone through the Suppression of the NF-kappaB and MAPK Signaling Pathways. Cells 2019;8

51. Tang WC, Hsu YC, Wang CC, Hu CY, Chio CC and Kuo JR. Early electroacupuncture treatment ameliorates neuroinflammation in rats with traumatic brain injury. BMC Complement Altern Med 2016;16:470

52. Tian GH, Tao SS, Chen MT, Li YS, Li YP, Shang HC, Tang XY, Chen JX and Tang HB. Electroacupuncture Treatment Alleviates Central Poststroke Pain by Inhibiting Brain Neuronal Apoptosis and Aberrant Astrocyte Activation. Neural Plast 2016;2016:1437148

53. Verboom L, Martens A, Priem D, Hoste E, Sze M, Vikkula H, Van Hove L, Voet S, Roels J, Maelfait J, Bongiovanni L, de Bruin A, Scott CL, Saeys Y, Pasparakis M, Bertrand MJM and van Loo G. OTULIN Prevents Liver Inflammation and Hepatocellular Carcinoma by Inhibiting FADD- and RIPK1 KinaseMediated Hepatocyte Apoptosis. Cell reports 2020;30:2237-2247 e6

54. Wang L, Xiong X, Zhang X, Ye Y, Jian Z, Gao W and Gu L. Sodium Tanshinone IIA Sulfonate Protects Against Cerebral Ischemia-reperfusion Injury by Inhibiting Autophagy and Inflammation. Neuroscience 2020

55. Xie C, Gao X, Luo Y, Pang Y and Li M. Electroacupuncture modulates stromal cell-derived factor1alpha expression and mobilization of bone marrow endothelial progenitor cells in focal cerebral 
ischemia/reperfusion model rats. Brain Res 2016;1648:119-126

56. Xu H, Mu S and Qin W. Microglia TREM2 is required for electroacupuncture to attenuate neuroinflammation in focal cerebral ischemia/reperfusion rats. Biochem Biophys Res Commun 2018;503:3225-3234

57. Xu H, Qin W, Hu X, Mu S, Zhu J, Lu W and Luo Y. Lentivirus-mediated overexpression of OTULIN ameliorates microglia activation and neuroinflammation by depressing the activation of the NFkappaB signaling pathway in cerebral ischemia/reperfusion rats. Journal of neuroinflammation 2018;15:83

58. Zhan J, Qin W, Zhang Y, Jiang J, Ma H, Li Q and Luo Y. Upregulation of neuronal zinc finger protein A20 expression is required for electroacupuncture to attenuate the cerebral inflammatory injury mediated by the nuclear factor-kB signaling pathway in cerebral ischemia/reperfusion rats. Journal of neuroinflammation 2016;13:258

59. Zhang Q, Wang Y, Ji G, Cao F, Hu G, Cong D, Xu X and Song B. Standardization of rehabilitation program for post-apoplectic limb spasm treated by Tongjing Tiaoxing tuina and scalp acupuncture with physical therapy. Medicine 2020;99:e20368

60. Zhang Q, Wei EQ, Zhu CY, Zhang WP, Wang ML, Zhang SH, Yu YP and Chen Z. Focal cerebral ischemia alters the spatio-temporal properties, but not the amount of activity in mice. Behav Brain Res 2006;169:66-74

61. Zhang R, Wu Y, Xie F, Zhong Y, Wang Y, Xu M, Feng J, Charish J, Monnier PP and Qin X. RGMa mediates reactive astrogliosis and glial scar formation through TGFbeta1/Smad2/3 signaling after stroke. Cell Death Differ 2018;25:1503-1516

62. Zhang W, Potrovita I, Tarabin V, Herrmann O, Beer V, Weih F, Schneider A and Schwaninger M. Neuronal activation of NF-kappaB contributes to cell death in cerebral ischemia. Journal of cerebral blood flow and metabolism : official journal of the International Society of Cerebral Blood Flow and Metabolism 2005;25:30-40

63. Zhang X, Zhao HH, Li D and Li HP. Neuroprotective effects of matrix metalloproteinases in cerebral ischemic rats by promoting activation and migration of astrocytes and microglia. Brain Res Bull 2018

64. Zhou Q, Yu X, Demirkaya E, Deuitch N, Stone D, Tsai WL, Kuehn HS, Wang H, Yang D, Park YH, Ombrello AK, Blake M, Romeo T, Remmers EF, Chae JJ, Mullikin JC, Guzel F, Milner JD, Boehm M, Rosenzweig SD, Gadina M, Welch SB, Ozen S, Topaloglu R, Abinun M, Kastner DL and Aksentijevich I. Biallelic hypomorphic mutations in a linear deubiquitinase define otulipenia, an early-onset autoinflammatory disease. Proceedings of the National Academy of Sciences of the United States of America 2016;113:10127-32

65. Zhu MY, Chen XQ, Huang F and Peng YX. [Effect of electroacupuncture on diaphragmatic function in patients with post-stroke tracheotomy]. Zhongguo zhen jiu = Chinese acupuncture \& moxibustion 2020;40:239-42 
Figures

A

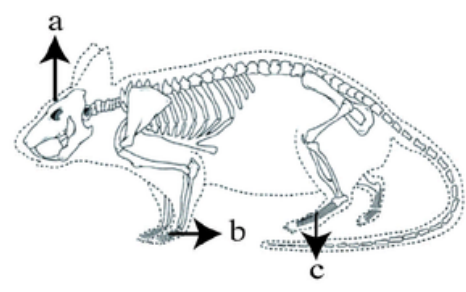

B

OTULIN expression

(RT-qPCR, Western blot and immunofluorescence)

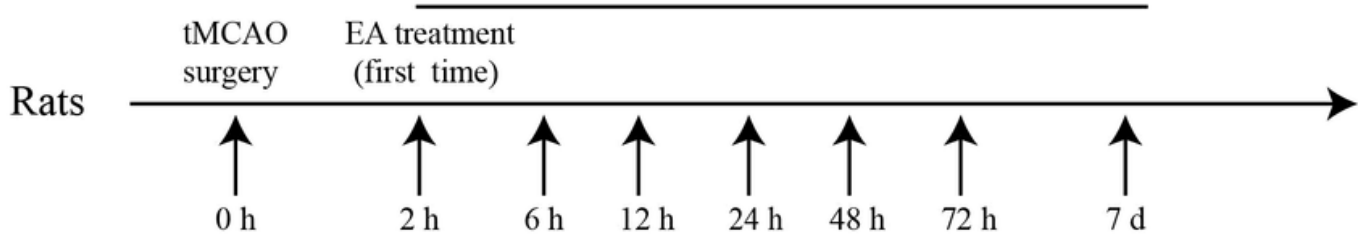

\section{Ischaemia \\ Reperfusion}

mNSS, inclined board test, rotarod test

TTC, immunofluorescence, TUNEL,

ICV injection of LV-shOTULIN or LV-Scramble

tMCAO

surgery

Nissl staining, Western blot, RT-qPCR

Rats

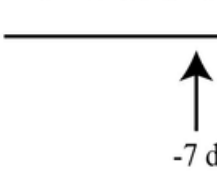

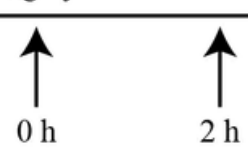

Ischaemia

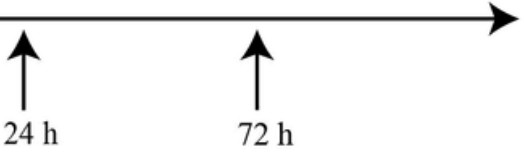

Reperfusion

\section{Figure 1}

The timeline of the experiment. (A) EA was performed at the indicated acupoints, a: Baihui acupoint (GV 20); b: Hegu acupoint (LI 4) (the figure comes from the paper with PMID 27453543, and permission was obtained from the author.); $\mathrm{c}$ : Taichong acupoint (LR 3). (B) The characteristics of OTULIN expression were detected at $6 \mathrm{~h}, 12 \mathrm{~h}, 24 \mathrm{~h}, 48 \mathrm{~h}, 72 \mathrm{~h}$, and $7 \mathrm{~d}$ after reperfusion. (C) The effects of OTULIN on the neuroprotective, neuroinflammatory and neuronal injury roles of EA were explored. 
A

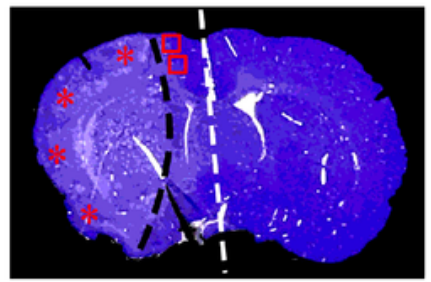

Ipsilateral

Contralateral

C

B

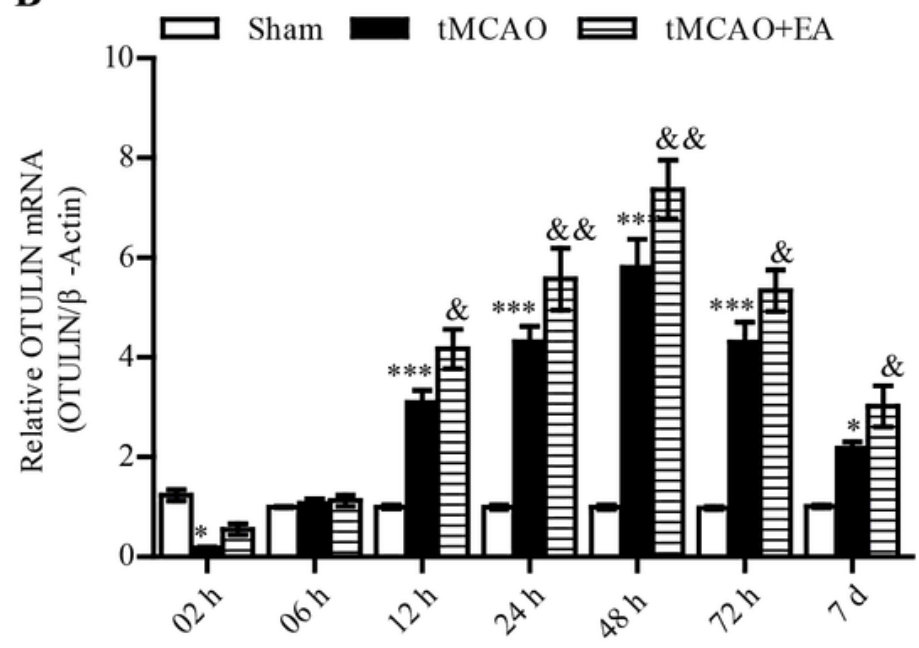

$\mathbf{E}$

OTULIN

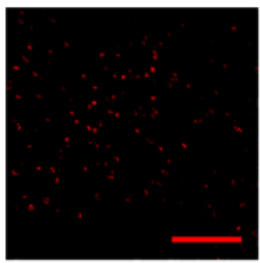

TMCAO
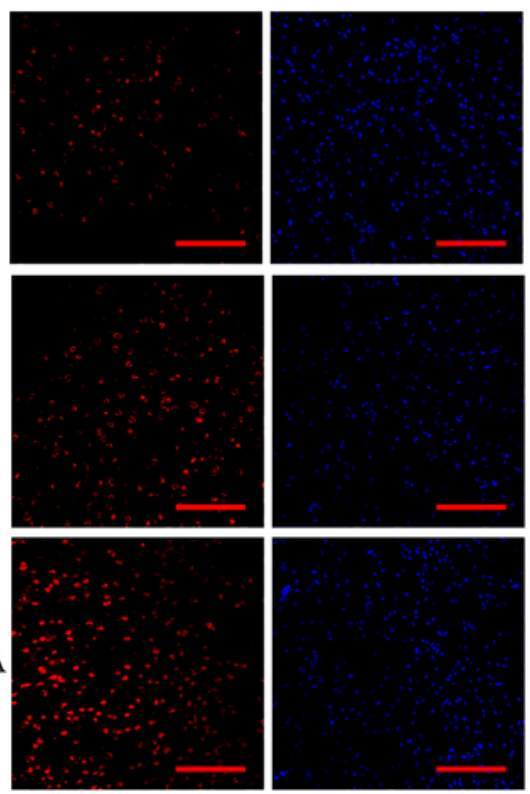

tMCAO+EA

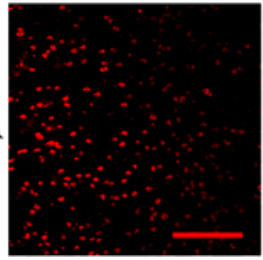

Sham

TMCAO

TMCAO+EA

$\beta$-Actin

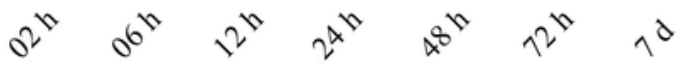

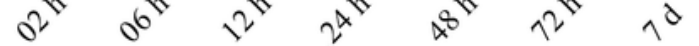

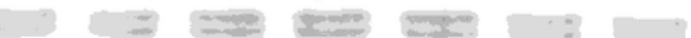

$\underline{2}$

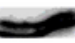

$=$

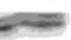

\section{D}

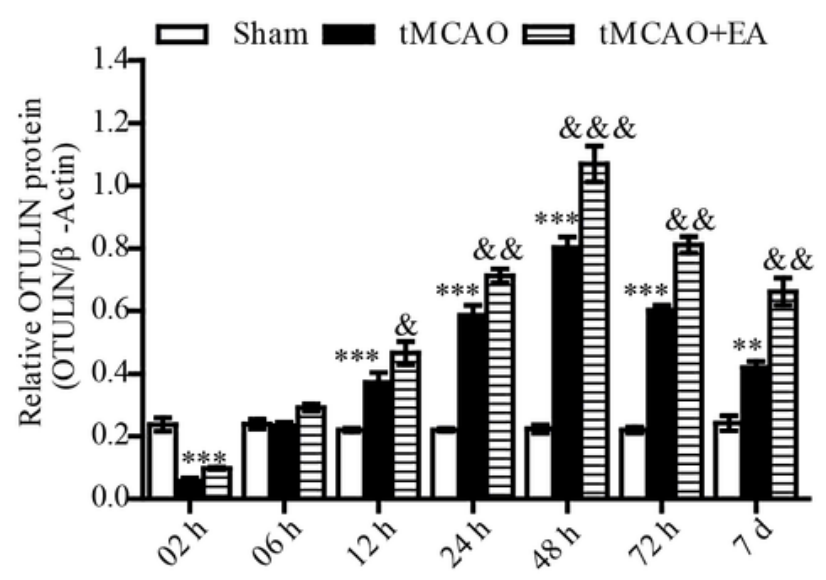

F
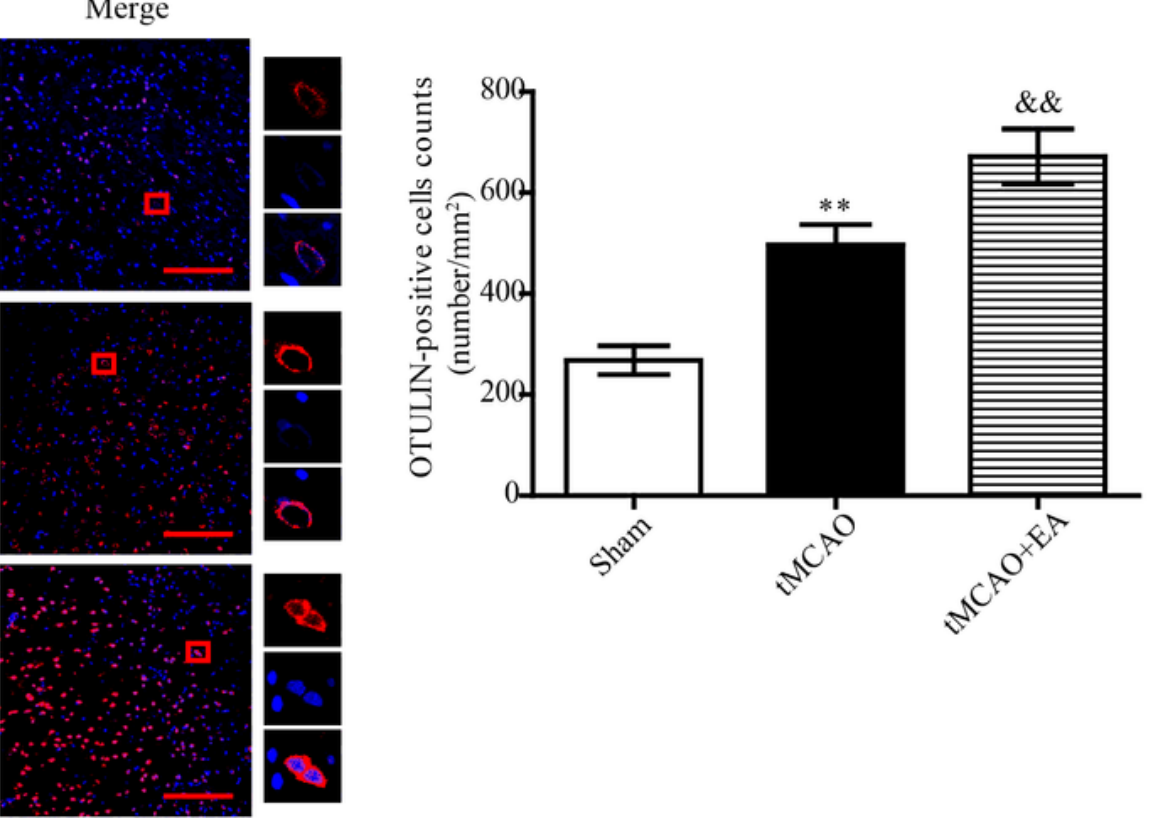

Figure 2

EA enhanced OTULIN expression in ischaemic stroke rats. (A) A coronal brain section was stained with cresyl violet to present the analysed regions (*: ischaemic core area; $\square$ : the ischaemic penumbra of the cerebral cortex; the white dotted line separated the ipsilateral and contralateral cerebral hemisphere.). (B) OTULIN mRNA levels at each time point were detected by RT-qPCR $(n=3)$. (C) OTULIN protein levels in each group were examined by Western blotting. (D) The histogram presents the quantitative analysis of 
OTULIN protein $(n=3)$. (E) Immunofluorescence for OTULIN expression in the cortical penumbra was performed at $48 \mathrm{~h}$ after reperfusion. Scale bar $=100 \mu \mathrm{m}$. (F) The histogram presents the quantitative analysis of OTULIN-positive cell counts $(n=8) .{ }^{* *} P<0.001,{ }^{*} P<0.01$, and ${ }^{*} P<0.05$ versus Sham group; $\& \& \& P<0.001, \& \& P<0.01$, and $\& P<0.05$ versus tMCAO group.

A

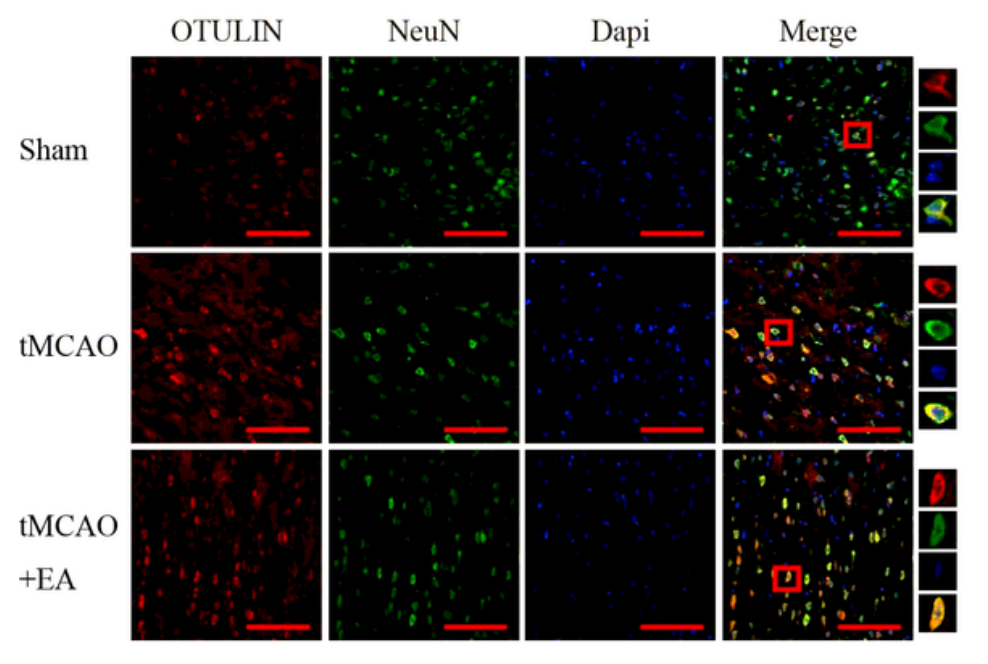

C

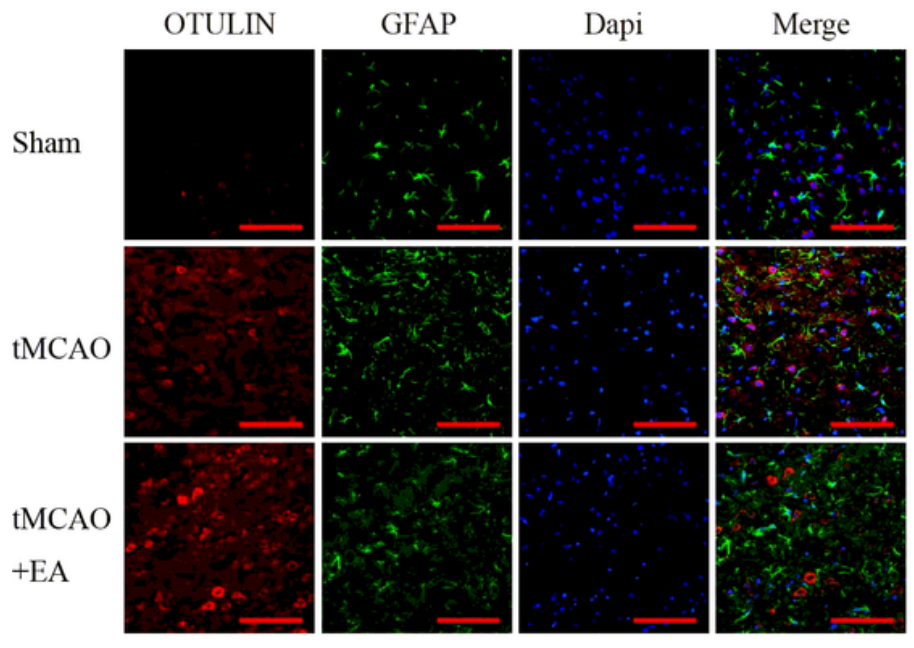

B

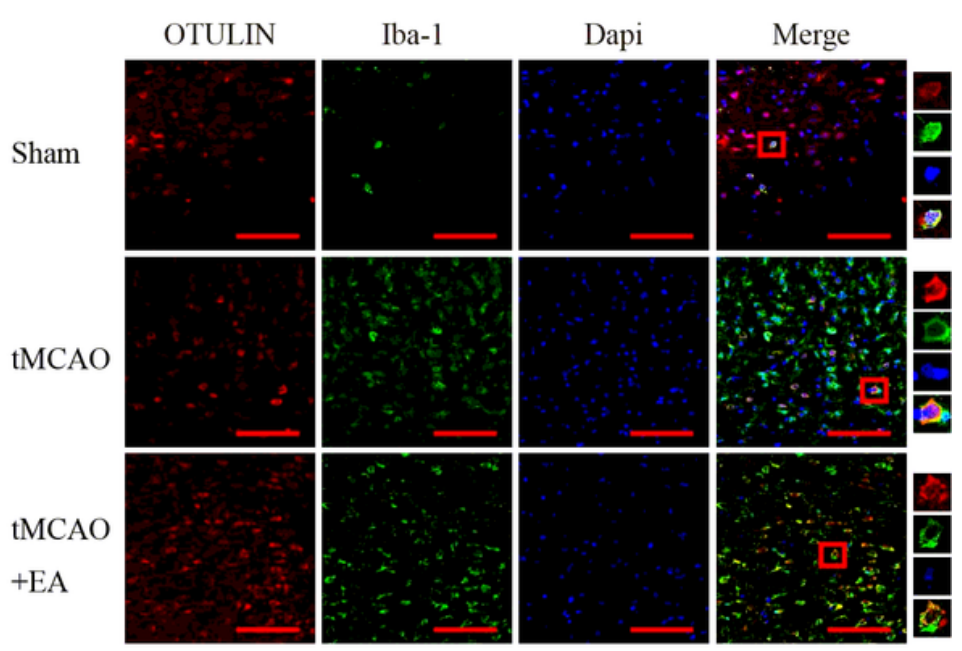

\section{Figure 3}

The cellular distribution of OTULIN in ischaemic stroke rats. OTULIN protein (red) was co-labelled with neurons (A green, identified by NeuN), microglia (B green, identified by Iba- 1 ), and astrocytes ( $\mathrm{C}$ green, identified by GFAP). The nucleus was stained with Dapi (blue). $n=8$ for each group. Scale bar $=100 \mu \mathrm{m}$. 

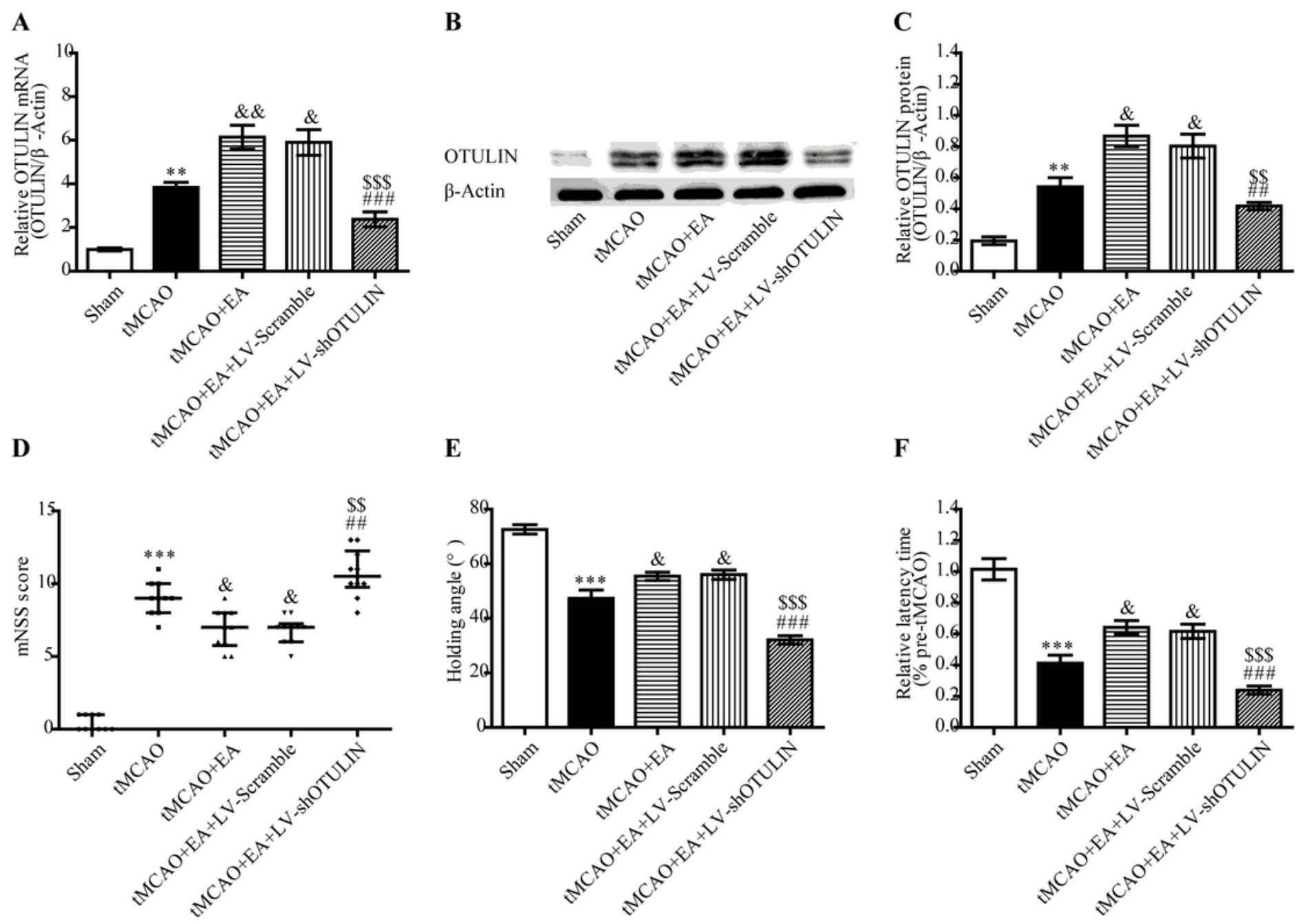

G

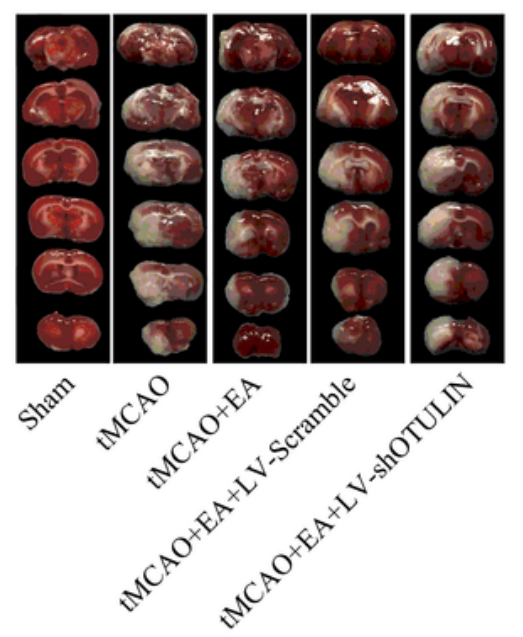

$\mathbf{H}$

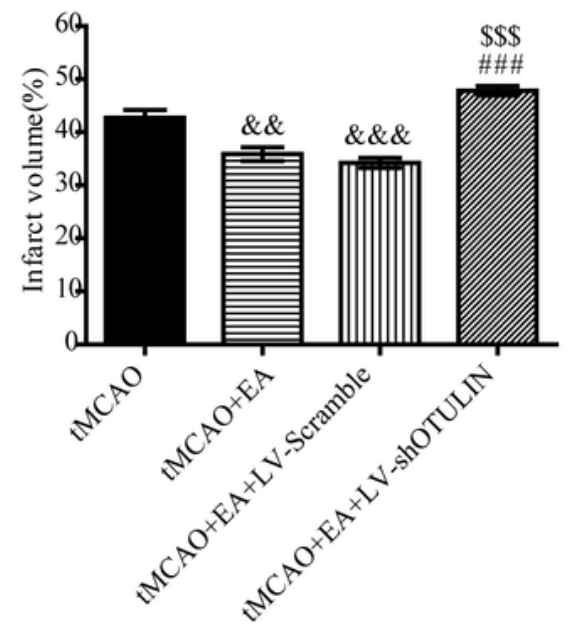

Figure 4

OTULIN silencing inhibited the neuroprotective effect of EA in focal cerebral ischaemia/reperfusion rats. Gene interference with OTULIN expression was assessed by RT-qPCR (A, n=3) and Western blotting (B and $C, n=3)$. Neurobehavioural function was evaluated using the mNSS score $(D, n=10)$, inclined board test $(E, n=10)$, and rotarod test $(F, n=10)$. (G) The graph shows an image of TTC (white: infarct area; red: 
noninfarct area). $(\mathrm{H}, \mathrm{n}=5)$ The panel presents the quantitative analysis of the cerebral infarct volume. ${ }^{* *} \mathrm{P}<0.001$ and ${ }^{* * P}<0.01$ versus Sham group; $\& \& \& P<0.001, \& \& P<0.01$, and $\& P<0.05$ versus tMCAO group;

$$
\$ P<0.001 \text { and }
$$

$\mathrm{P}<0.01$ versus tMCAO+EA group; \#\#\#P<0.001 and \#\#P<0.01 versus tMCAO+EA+LV-Scramble group.

A

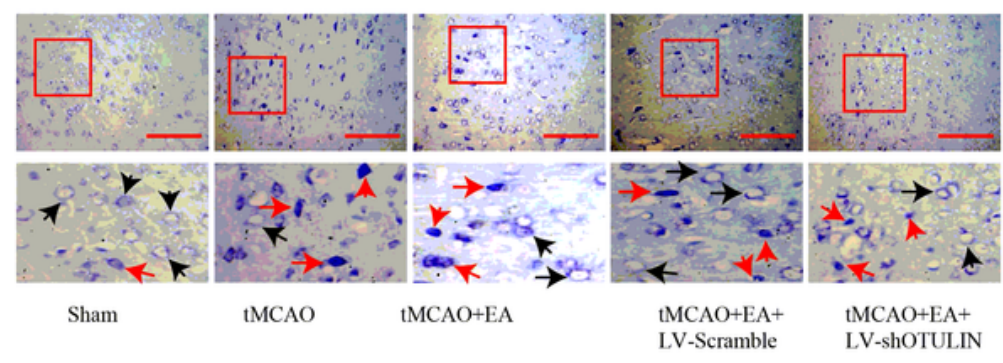

$\mathrm{C}$

Sham

IMCAO

MCAO+EA

MCAO+EA

+LV-Scramble

TMCAO+EA

+ LV-shOTULIN
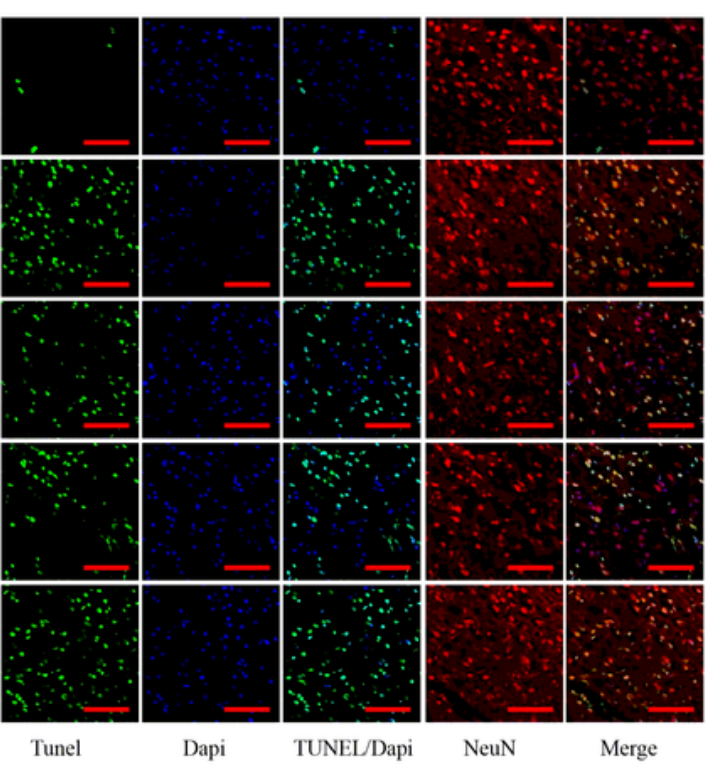

Dapi

TUNEL/Dapi

NeuN

Merge

F

Sham

MCAO

MCAO+EA

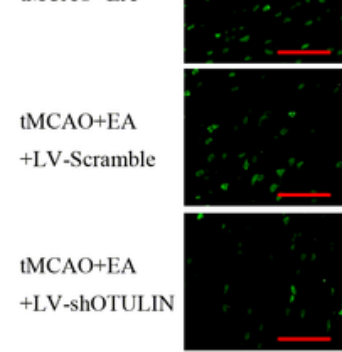

NeuN
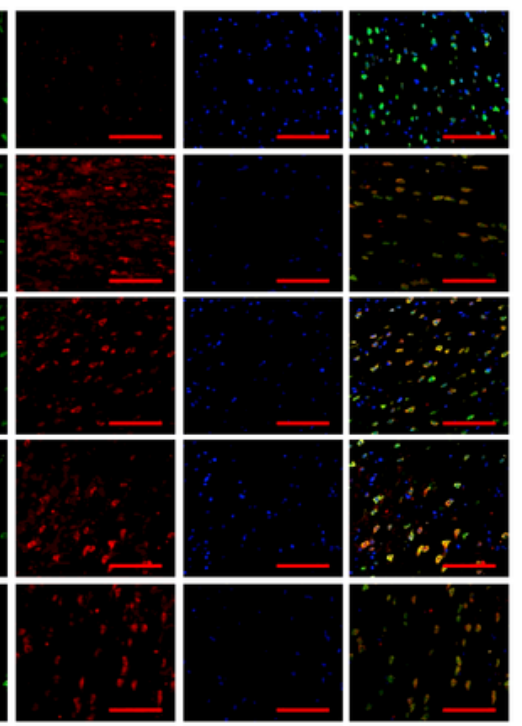

p65

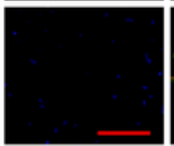

Dapi
B
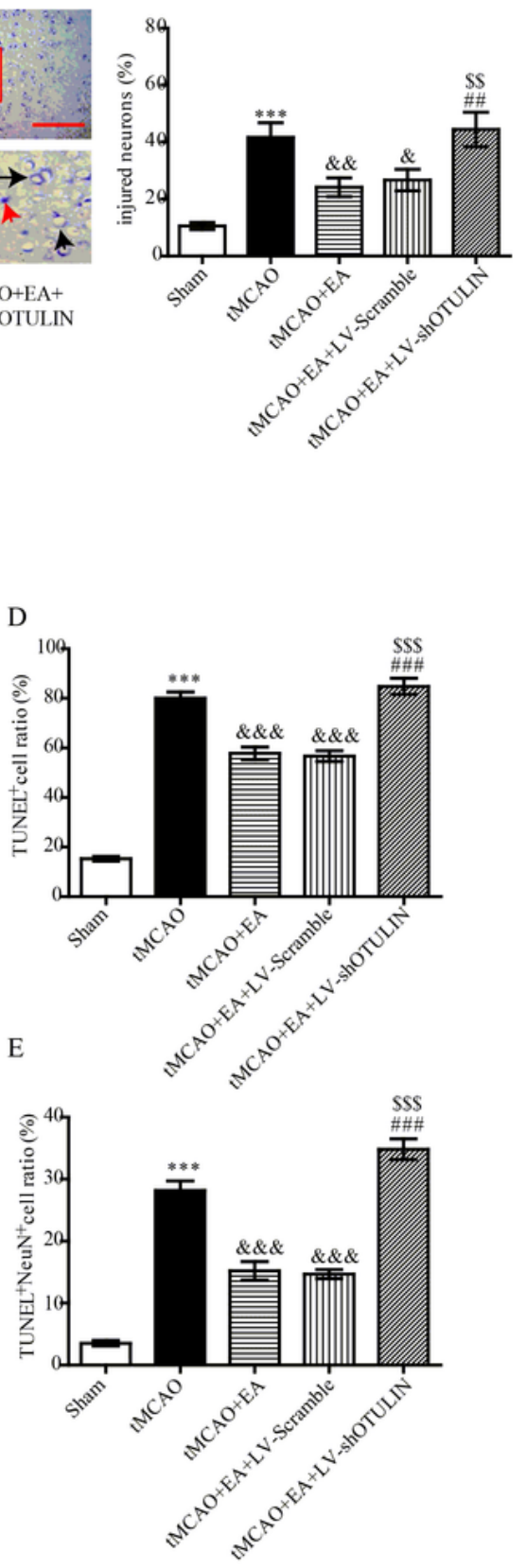

Figure 5 
OTULIN was required for EA to attenuate neuronal injury in focal cerebral ischaemia/reperfusion rats. (A) Nissl staining was used to observe the morphology of neurons in the ischaemic penumbra of the cerebral cortex. Black arrows and red arrows represent normal neurons and injured neurons, respectively. $(B, n=3)$ The statistical analysis of injured neurons by Nissl staining. (C) Immunofluorescence was used to detect TUNEL+ cells and TUNEL+NeuN+ cells. Panels $D(n=3)$ and $E(n=3)$ represent quantitative analysis of the TUNEL+ cells and TUNEL+NeuN+ cells. (F) NeuN and NF-KB p65 were co-stained to observe the nuclear translocation of neuronal NF-KB p65 by immunofluorescence. ${ }^{*} * \mathrm{P}<0.001$ versus Sham group; $\& \& \& P<0.001, \& \& P<0.01$, and $\& P<0.05$ versus tMCAO group;

$$
\$ P<0.001 \text { and }
$$

$P<0.01$ versus $t M C A O+E A$ group; \#\#\#P<0.001 and \#\#P<0.01 versus $t M C A O+E A+L V-S c r a m b l e ~ g r o u p$. Scale bar $=100 \mu \mathrm{m}$. 
A

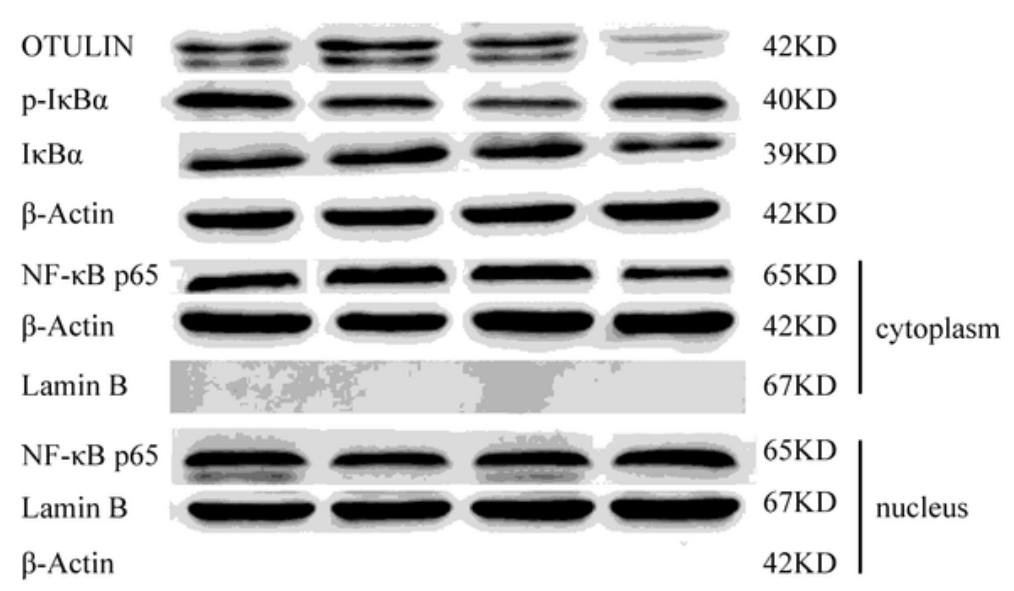

$\beta$-Actin

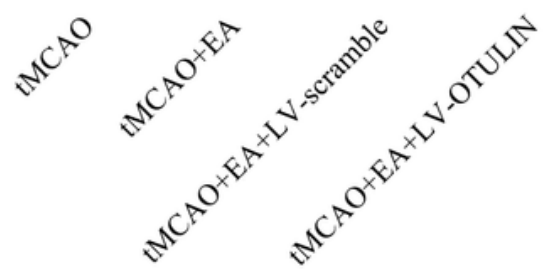

B

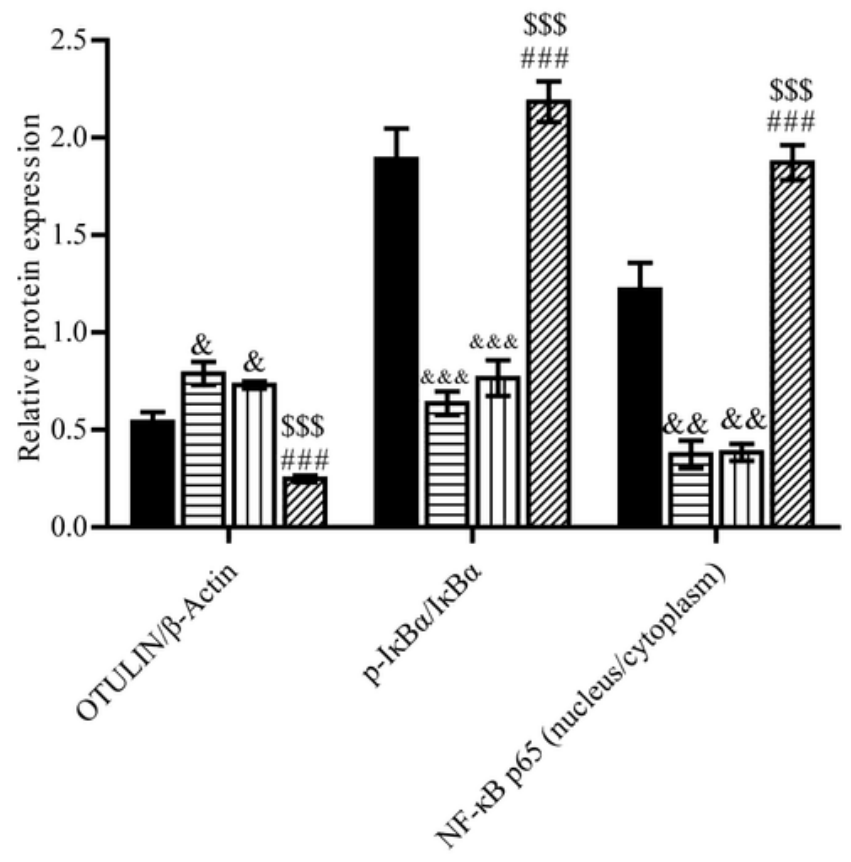

C

$\mathrm{I} \kappa \mathrm{B} \alpha$
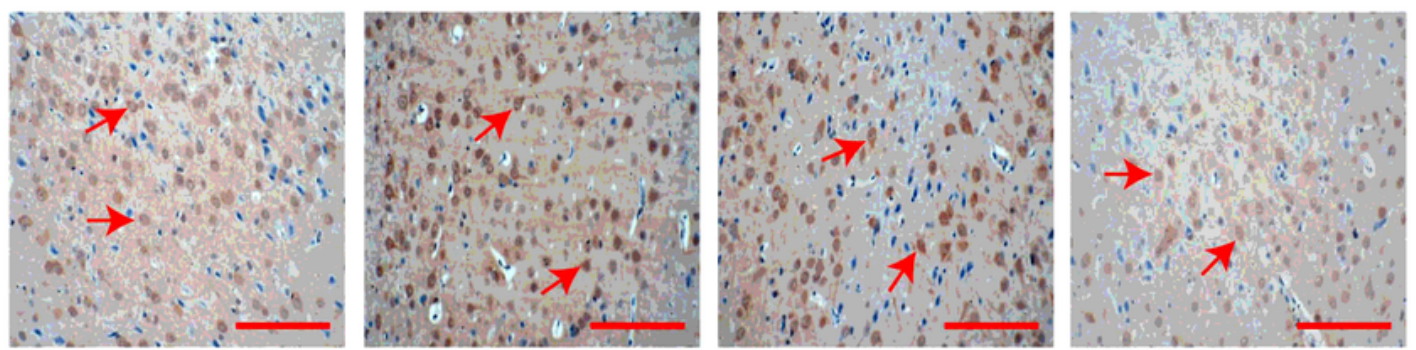

NF-kB p65
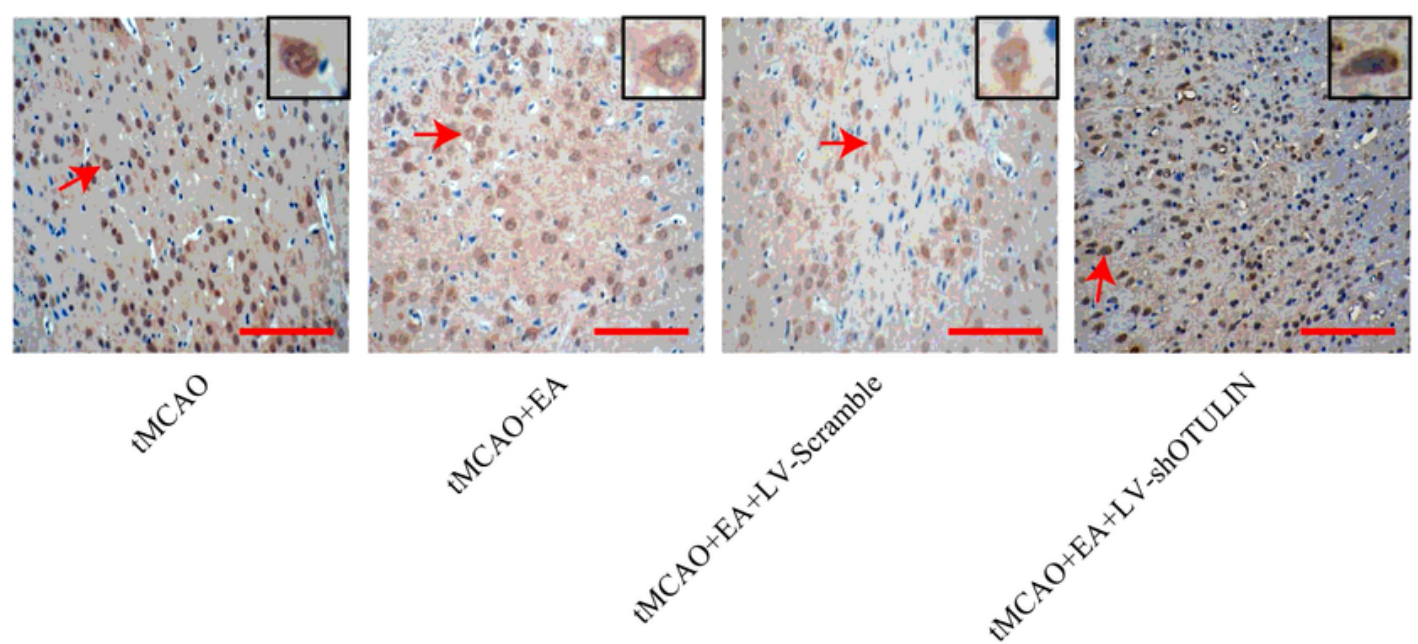

\section{Figure 6}

EA inhibited the NF-KB signalling pathway by upregulating OTULIN expression. (A) Western blotting was used to detect OTULIN, p-ІкBa, ІкBa, and cytoplasm/nucleus-p65 protein. $\beta$-Actin served as a loading control for total or cytoplasmic protein, and Lamin B served as a nuclear protein. (B, $n=3)$ Quantitative analysis of OTULIN protein, the phosphorylation ratio of ІкBa, and nuclear/cytoplasmic NF-KB p65. (C, $\mathrm{n=6)}$ Immunohistochemistry was performed to detect ІкBa and NF-kB p65 protein. Red arrows refer to 
IkBa- or NF-KB p65-positive cells. Scale bar $=100 \mu \mathrm{m} . \& \& \& \mathrm{P}<0.001, \& \& \mathrm{P}<0.01$ and $\& \mathrm{P}<0.05$ versus tMCAO group; $\$ \$ P<0.001$ versus tMCAO+EA group; \#\#\#P<0.001 versus tMCAO+EA+LV-Scramble group.

A Aa
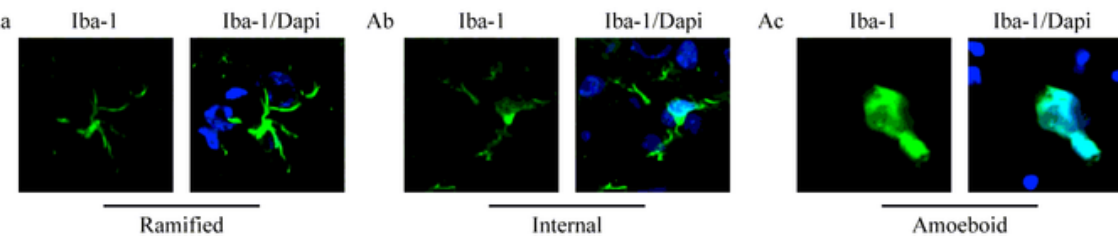

C

D
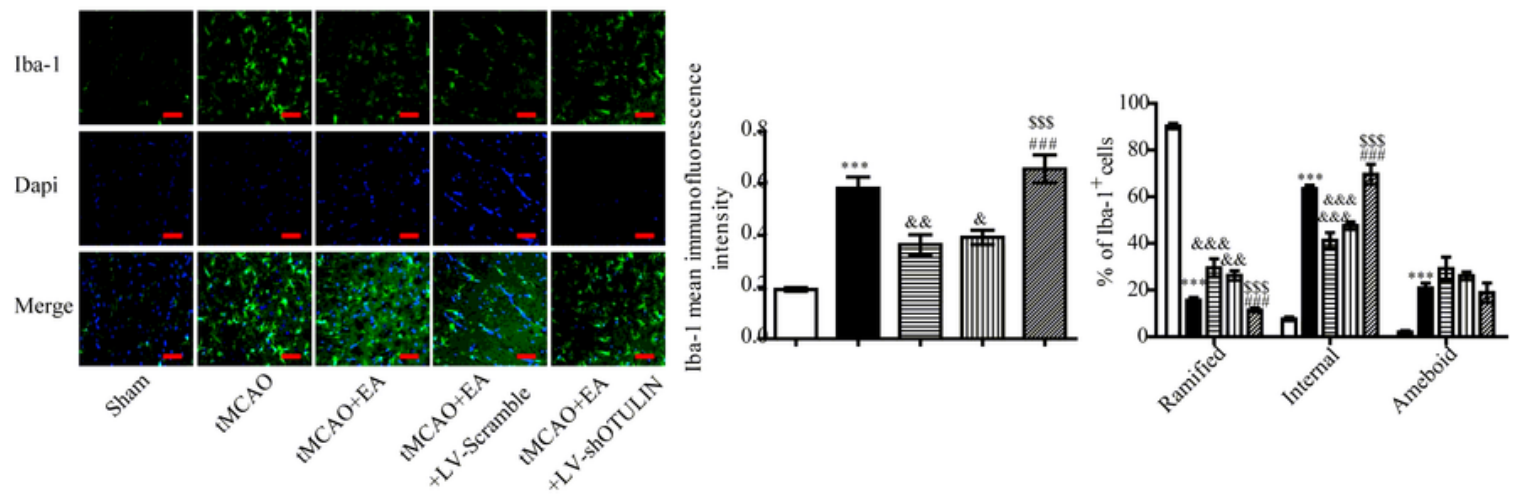

E

$\mathrm{F}$

G

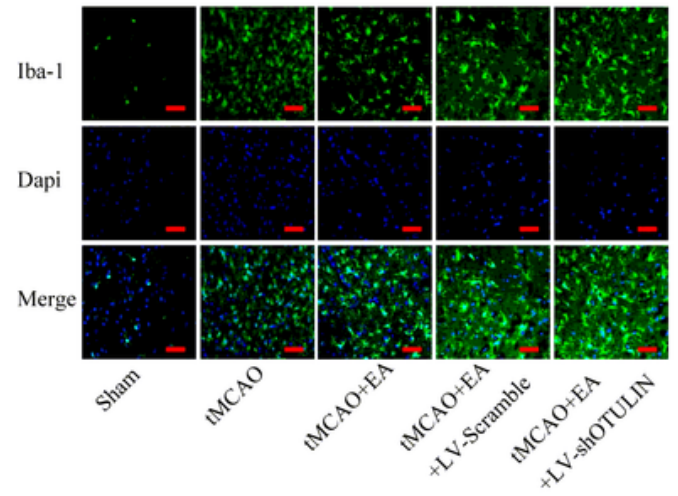

$\mathrm{H}$

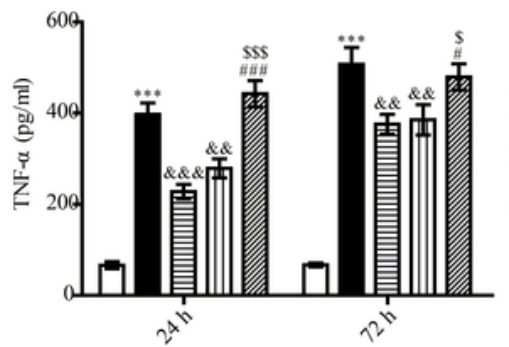

$\mathrm{K}$

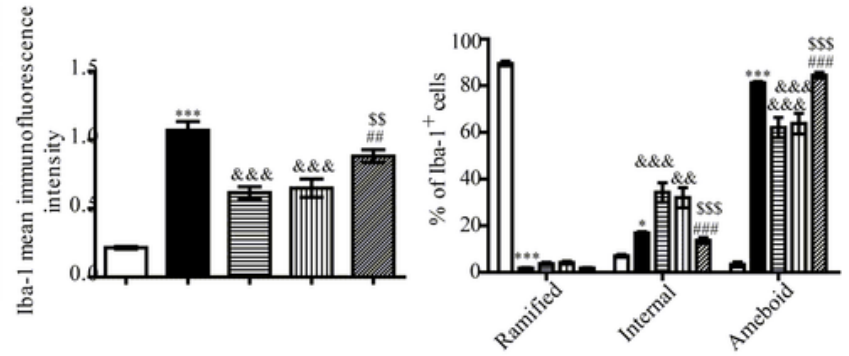

$\mathrm{J}$

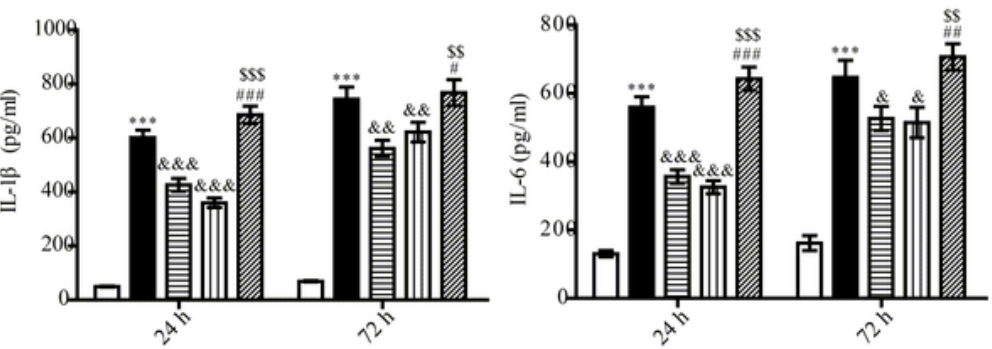

tMCAO

Figure 7

OTULIN silencing weakened the inhibitory effect of EA on microglial activation in focal cerebral ischaemia/reperfusion rats. (A) Representative images of Iba- $1+$ cells in the ischaemic penumbra of the cerebral cortex: (Aa) Ramified cells; (Ab) Internal cells; (Ac) Ameboid cells. The Iba-1+ microglial cells in 
the ischaemic penumbra of cerebral ischaemia were detected by immunofluorescence at $24 \mathrm{~h}(\mathrm{~B})$ and 72 $h(E)$ after reperfusion. Histograms $C(n=6)$ and $F(n=6)$ represent the quantitative analysis of mean Iba-1 immunofluorescence intensity at $24 \mathrm{~h}$ and $72 \mathrm{~h}$, respectively. Histograms $D(n=6)$ and $G(n=6)$ represent the quantitative analysis of the proportions of ramified, internal, and ameboid cells at $24 \mathrm{~h}$ and $72 \mathrm{~h}$, respectively. The contents of TNF-a $(H, n=6), I L-1 \beta(I, n=6)$ and IL-6 $(J, n=6)$ at $24 h$ and $72 h$ were detected. Scale bar $=50 \mu \mathrm{m}$. ${ }^{* *} \mathrm{P}<0.001$ and ${ }^{*} \mathrm{P}<0.05$ versus Sham group; $\& \& \& P<0.001, \& \& P<0.01$, and $\& P<0.05$ versus $\mathrm{tMCAO}$ group;

$$
\$ P<0.001,
$$

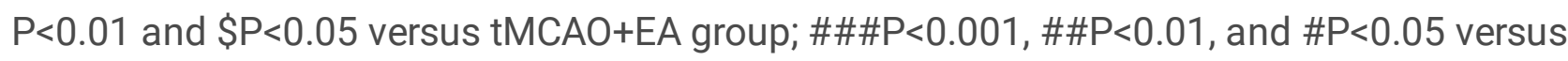
tMCAO+EA+LV-Scramble group. 
A

$24 \mathrm{~h}$

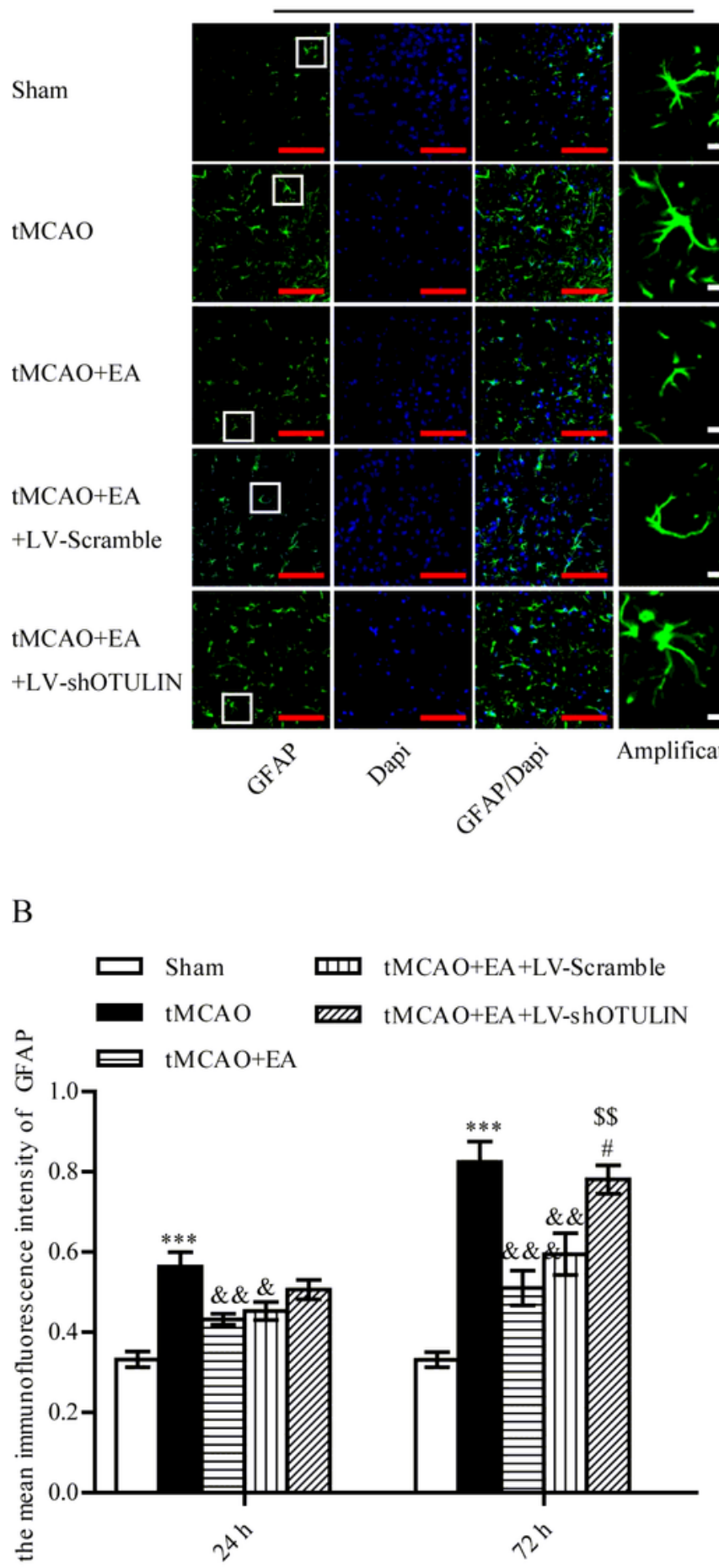

$72 \mathrm{~h}$

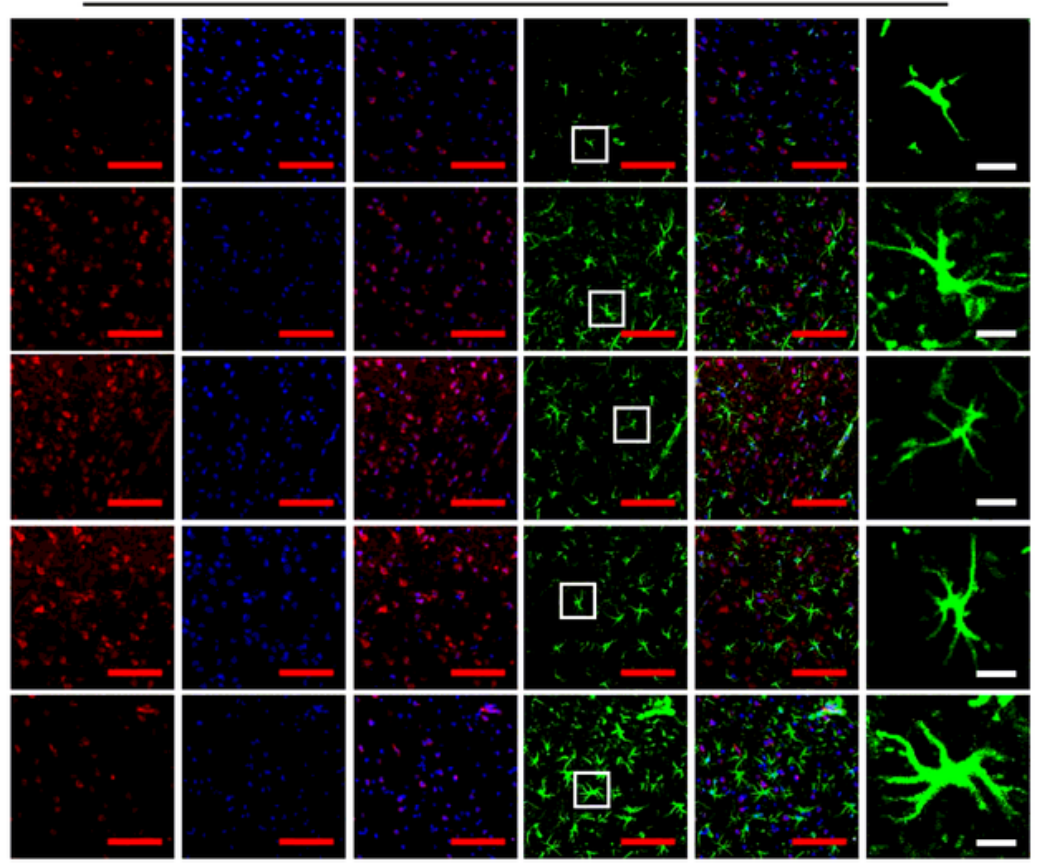

Amplification

C
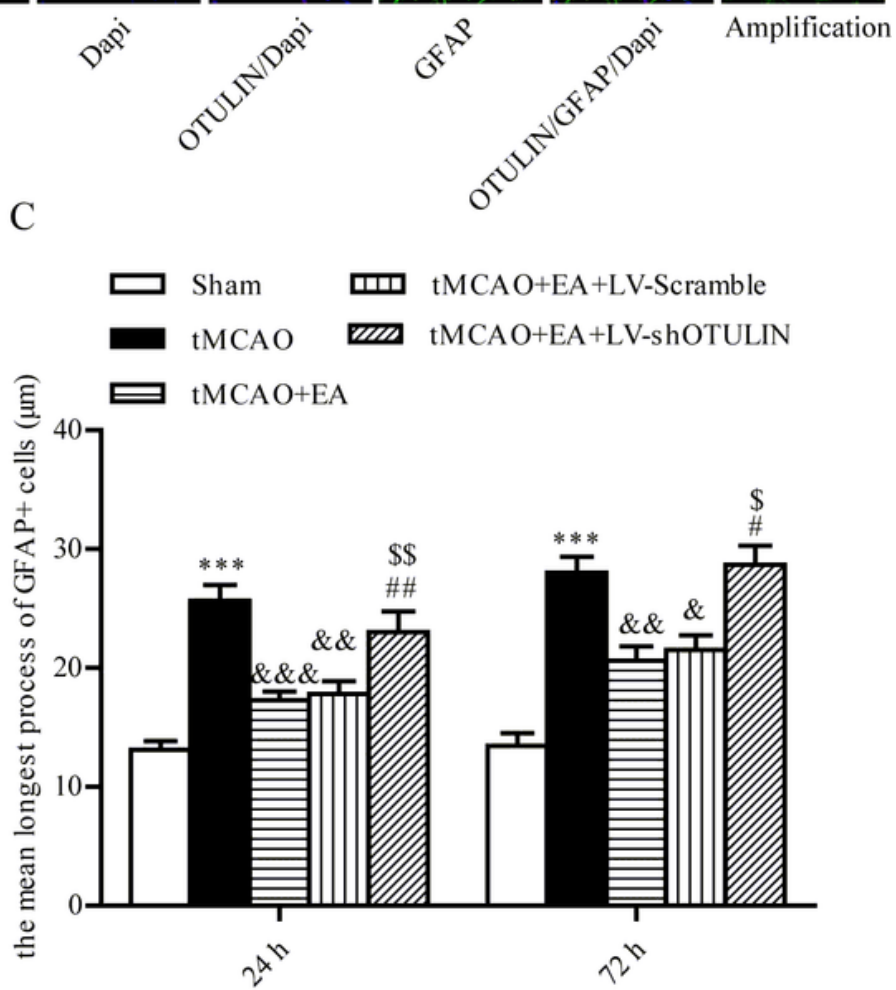

Figure 8

OTULIN was involved in EA's role in attenuating astrogliosis in focal cerebral ischaemia/reperfusion rats.

(A) Immunofluorescence was used to stain astrocytes at $24 \mathrm{~h}$ and $72 \mathrm{~h}$. $(B, n=6)$ The mean

immunofluorescence intensity of GFAP at $24 \mathrm{~h}$ and $72 \mathrm{~h}$ was analysed quantitatively. $(\mathrm{C}, \mathrm{n}=6)$ The mean longest process of GFAP+ cells was quantitatively analysed. ${ }^{\star} \star * P<0.001$ versus Sham group; 
$\& \& \& \mathrm{P}<0.001, \& \& \mathrm{P}<0.01, \& \mathrm{P}<0.05$ versus $\mathrm{tMCAO}$ group; $\$ \mathrm{P}<0.01$ and $\$ \mathrm{P}<0.05$ versus $\mathrm{tMCAO}+\mathrm{EA}$ group; $\# P<0.05$ versus tMCAO+EA+LV-Scramble group. Scale bar $($ red $)=100 \mu \mathrm{m}$, Scale bar (white) $=10 \mu \mathrm{m}$. 